Gesnerus 52 (1995) 209-246

\title{
Dürrenmatt und die Naturwissenschaften
}

Hans Wolfgang Bellwinkel

\begin{abstract}
Himmel und Erde, Welt und Gestirn gehören für einen Schriftsteller untrennbar zusammen, dessen poetische Absicht zeitlebens der Veranschaulichung des kopernikanischen Bewusstseins galt.

Walter Jens: Gedenkrede bei der Trauerfeier für F. D. in Bern'
\end{abstract}

\section{Summary}

The natural sciences, especially physics, mathematics, cosmology and in the last years, biology, play a very important role in the literary and pictorial work of Friedrich Dürrenmatt. He not only deals with them in a critical way, but they also characterize his way of thinking. In this way Dürrenmatt combines the two cultures, humanities and natural sciences, in a fruitful synthesis.

Frau Charlotte Kerr-Dürrenmatt, Herrn Prof. Dr. Marc Eichelberg, Herrn Ueli Weber (Dürrenmatt-Archiv), Frau Dorothee Dutler, Frau Vera Heuberger, Frau Uta Kunau, Herrn Dr. phil. Klaus E. Bohnenkamp, und Herrn Martin Bellwinkel danke ich für ihre Unterstützung durch zahlreiche Hinweise, Informationen und kritische Stellungnahmen sehr herzlich. Mein Dank gilt auch Herrn Prof. A. Dress vom Mathematischen Institut der Universität Bielefeld, der mich in mathematischen Fragen beraten hat.

Aus Platzgründen wurde das Kapitel über das bildnerische Werk, das in der ausführlichen Version der Arbeit enthalten ist, von der Redaktion gestrichen.

Die Dürrenmatt-Zitate sind entnommen aus:

Dürrenmatt, F.: Gesammelte Werke in sieben Bänden. Zürich: Diogenes, 1991;

Dürrenmatt, F.: Gedankenfuge. Zürich: Diogenes, 1992;

Dürrenmatt, F.: Über die Grenzen. München/Zürich: R. Piper, 1993.

1 F. D. Schriftsteller und Maler. Hrsg.: Schweizerisches Literaturarchiv, Bern, und Kunsthaus Zürich, 1994; Ausstellungskatalog, S. 263.

Dr. med. Hans Wolfgang Bellwinkel, Erlenstrasse 8, D-44795 Bochum 


\section{Zusammenfassung}

Die Naturwissenschaften spielen in Dürrenmatts literarischem und bildnerischem Werk eine sehr wichtige Rolle, besonders die Physik, Mathematik, Kosmologie und in den letzten Jahren auch die Biologie. Sie werden nicht nur kritisch betrachtet, sondern prägen auch in entscheidendem Masse seine Denkweise. So verbindet Dürrenmatt die zweite, die naturwissenschaftliche Kultur mit den Geisteswissenschaften in fruchtbarer Synthese.

Friedrich Dürrenmatt (F. D.) gehört zu den wenigen deutschsprachigen Schriftstellern, die sich intensiv mit der modernen Naturwissenschaft beschäftigt und diese in ihr Werk integriert haben. In einem Interview mit Sven Michaelsen am 7.12.90, kurz vor seinem Tod $^{2}$, begründet er diese Feststellung:

«... die Welt dramaturgisch in den Griff zu bekommen, das geht heute ohne die Beschäftigung mit der (Natur-)Wissenschaft überhaupt nicht. Was die Welt verändert, ist doch ... eben die (Natur-)Wissenschaft. Die zweite, die naturwissenschaftliche Kultur ist heute das Entscheidende. Die Politik hinkt nach.»

Ein Grundmotiv seiner intensiven Beschäftigung mit den Naturwissenschaften im weitesten Sinne, die Mathematik eingeschlossen, ist die Faszination und Liebe zu diesem Wissenszweig, die er in einer Widmung für den Freund Marc Eichelberg im Buch F. D. «Bilder und Zeichnungen» zum Ausdruck bringt. Darin bezeichnet er sich als Amateur auf dem Gebiet der Naturwissenschaften; Amateur, unter Hinweis auf E. Friedell,im Sinne von Liebhaber.

Das bestätigt sich in der Art, wie er mit physikalischen und astronomischen Begriffen gedanklich spielt, sie als metaphorische Versatzstücke für seine politischen und gesellschaftspolitischen Szenarios benutzt. Das beweist aber ferner, dass er die Naturwissenschaften nicht nur kennt, sondern auch die grossen Zusammenhänge beherrscht. Dabei liegen die Schwerpunkte im Bereich der Kosmologie, Physik und Mathematik; weniger ausgeprägt ist die Beschäftigung mit der Biologie. Was die Analyse des Dürrenmattschen Oeuvres vom Standpunkt der Naturwissenschaften erschwert, ist die enge Verknüpfung mit der Philosophie, der ja auch sein Studium in Zürich und Bern gewidmet war. Daraus resultieren die vielen erkenntnis-theoretischen Überlegungen, die an verschiedenen Stellen in das Werk eingestreut sind.

Aufgabe dieser Arbeit ist es zu zeigen, dass das naturwissenschaftliche Denken in Dürrenmatts Werk nicht marginal ist, sondern sein ganzes Oeuvre durchtränkt, eine für ihn spezifische Denkweise darstellt.

2 F. D.: Über die Grenzen, S. 15. 
Will man dem Leser dieser Arbeit das Dürrenmattsche naturwissenschaftliche Denken deutlich vor Augen führen, die Schärfe und Brillanz seiner an Mathematik und Logik geschulten Formulierungen und die Bildhaftigkeit seiner Sprache, mit der es ihm gelingt, auch schwierigste Zusammenhänge zu erhellen, dann muss man immer wieder auf das wörtliche Zitat zurückgreifen. Das Original bringt eben mehr als die alleinige Interpretation.

\section{Quellen}

Wenn man sich, wie bei Dürrenmatt, einem so immensen naturwissenschaftlich-mathematischen Wissen gegenübersieht, stellt sich die Frage nach den Quellen, aus denen der Autor dieses Wissen schöpft. Im Gegensatz zu Thomas Mann, der in seinen akribisch geführten Tagebüchern viele Hinweise auf die Quellen seines Wissens gibt, hat F. D. nie ein Tagebuch geführt, ja das Führen eines Tagebuches sogar ausdrücklich abgelehnt ${ }^{3}$. Infolgedessen ist das Aufspüren seiner Quellen ungleich schwieriger. Autobiographische Bemerkungen finden sich weit verstreut in seinen Werken, besonders aber in den Stoffen I-III im Kapitel «Mondfinsternis».

Schon in seiner Kindheit und frühen Jugend in einem kleinen Dorf im Emmental, Kanton Bern, wurde ihm von einem seiner Lehrer die Sternenwelt nahegebracht. Mit 8 Jahren kannte der Knabe die Namen sämtlicher Sternbilder, die er mit seinem selbstgebastelten Fernrohr, häufig von einem Baumwipfel aus, beobachtete. In seinen Erinnerungen, die er 1968 unter dem Titel «Dokument» veröffentlichte, beschreibt er diese Zeit:

«Über den Wäldern stehen die Sterne. Ich machte mit ihnen früh Bekanntschaft, zeichnete ihre Konstellationen, den unbeweglichen Polarstern, den kleinen und den grossen Bären mit dem geringelten Drachen zwischen ihnen, ich lernte die helle Wega kennen, den funkelnden Atair, den nahen Sirius, die ferne Deneb, die Riesensonne Aldebaran, die noch gewaltigeren Betageuze und Antares. Ich wusste, dass das Dorf zur Erde und die Erde zum Sonnensystem gehöre, dass die Sonne mit ihren Planeten sich um das Zentrum der Milchstrasse bewegte, Richtung Herkules, und ich vernahm, dass der gerade noch vom blossen Auge erkennbare Andromeda-Nebel eine Milchstrasse sei wie die unsrige. Ich war nie ein Ptolemäer.»

Hier begann seine grosse Liebe zu und sein Interesse an den Naturwissenschaften, die zeitlebens anhielt.

- In «Persönliche Anmerkungen zu meinen Bildern und Zeichnungen», $1978^{5}$, sagt er Ähnliches. In Neuchâtel hat er sich ein grosses Teleskop zugelegt, von dem er unter «Autobiographisches», 1980/83 Vallon de l'Ermitage ${ }^{6}$ berichtet:

3 F. D.: Ebenda, S. 15.

4 In: Ausstellungsschrift des Heinrich-Heine-Institutes, Düsseldorf, 1993, S. 1.

5 Bd. 7, S. $507 . \quad 6$ Bd. 7, S. 929. 
«Aber meistens verwende ich den Zeiss»

- ein grosses zweirohriges Fernrohr auf einem Stativ, das noch heute im grossen Wohn- und Arbeitszimmer seines Hauses in Neuchâtel nahe dem Schreibtisch steht -,

«um den Mond und die Planeten zu beobachten. Jupiter und Saturn sehe ich darin wie gestochen. Für die Jagd auf Spiralnebel setze ich ein zweiundzwanzig-Zentimeter-Spiegelteleskop ein...»

Noch 1986 sagte F. D. in einem Gespräch mit Franz Kreuzer:

«Ich lese fast nur wissenschaftliche und philosophische Bücher»

und wenige Tage vor seinem Tode zu Sven Michaelsen:

«(Ich lese) Meist Naturwissenschaftliches und Philosophisches. Heute Nacht ein Buch über ungelöste mathematische Probleme.» ${ }^{7}$

Um so erstaunlicher ist es, dass er auf der höheren Schule jahrelang den Physik-Unterricht geschwänzt hat. Kurz vor der Maturität hat er zusammen mit einem Freund dieses Defizit zu beseitigen versucht. Die beiden holten in 2monatigem intensiven Studium unter der Anleitung eines jungen Physikers auf, was sie zuvor versäumt hatten.

«Die Physik begann mich zu faszinieren: zum ersten Mal ahnte ich, was exakte Begriffe bedeuten: nicht eine Wahrheit, die gänzlich unabhängig vom Menschen wäre, im Gegenteil, aber eine Wahrheit, die es nur dank der Vernunft gibt ... eine 〈menschliche Wahrheit>... ${ }^{8}$

Hier schimmert das Gedankengut Alexander Wittenbergs durch, mit dessen Schrift «Vom Denken in Begriffen» sich F. D. intensiv auseinandergesetzt hat. Und wenige Seiten weiter heisst es:

«Eine Vorliebe für die Physik hat mich seitdem nicht mehr verlassen. Sie und die Mathematik sind die einzigen Fächer, von denen ich bedaure, sie in meiner Gymnasial- und Universitätszeit nicht fleissiger studiert zu haben. Die Erkenntnis, dass gerade sie für mich wichtig wären, kam zu spät, und nun bleibt mir nichts anderes übrig, als in ihnen zu stümpern. Wohl lese ich mathematische oder physikalische Bücher, doch vermag ich ihren Inhalt bloss zu ahnen.»

Ein Blick auf Dürrenmatts Schreibtisch in seinem Neuchâteler Haus ist sehr aufschlussreich. Neben verschiedenen Duden-Ausgaben finden sich sozusagen als Handbibliothek Talbot: Mystik und Physik; St. Körner: Philosophie der Mathematik; R. Kassner: Zahl und Gesicht. In seiner SchlafzimmerBibliothek stehen u.a.: K. Popper und J. C.: Eccles: Das Ich und sein Gehirn; Dawkins: Das egoistische Gen; François Fricker: Einführung in die Gitterpunktlehre.

Eine ganz entscheidende Rolle für Dürrenmatts Weiterbildung in den Na-

7 Über die Grenzen, S. 14.

8 Stoffe I-III, «Mondfinsternis», Bd. 6, S. 200. 
turwissenschaften spielte sein langjähriger Freund Marc Eichelberg, zuletzt Professor für Physik und Mathematik an der Lehrerbildungsanstalt in Chur. Während dessen Neuchâteler Zeit hat er 2 Monate lang einmal wöchentlich mit F. D. über das Buch des Mathematikers Alexander Wittenberg:Vom Denken in Begriffen. Mathematik als Experiment des reinen Denkens ${ }^{9}$, diskutiert. Eichelberg war es auch, der seinem Freund die grundlegenden Bücher auf dem Gebiet der Mathematik, Naturwissenschaft und Naturphilosophie empfohlen hat. Die wichtigsten Titel sind: G. Vollmer: Evolutionäre Erkenntnistheorie; Hermann Weyl: Philosophie der Mathematik und Naturwissenschaft; Hermann Bondi: Einsteins Einmaleins; Heinrich Tietze: Gelöste und ungelöste mathematische Probleme;Alfred N. Whitehead: Die Funktion der Vernunft - um nur einige zu nennen. In «Nachträgliche Vorbemerkungen» zu dem Vortrag über Toleranz ${ }^{10}$ erwähnt F. D. noch A. Eddington: Philosophie der Naturwissenschaften; Immanuel Kant und Karl Popper. In den Anmerkungen zu Albert Einstein ${ }^{11}$ finden sich weitere Hinweise auf Autoren wie C. B. Collins, Stephen W. Hawking, Rudolf Carnap, H. Poincare, B. Hoffmann, Res Jost und K. Gödel. In einem Brief vom 22.9.82 erklärt Marc Eichelberg seinem Freund F. D. einige Grundbegriffe der Physik wie Masse, Kraft, Arbeit, Energie und Leistung (ausgestellt im Heinrich-Heine-Institut in Düsseldorf, 1993). Auch der Vortrag «Albert Einstein», 1979 von F. D. an der ETH Zürich gehalten ${ }^{12}$, wurde in enger Zusammenarbeit mit seinem Freund konzipiert. Noch am Tage vor seinem Tode bekam F. D. Besuch von Marc Eichelberg. Sie wollten gemeinsam am nächsten Tag den Essay «Kabbala der Physik» durchsprechen. Dazu kam es nicht mehr; denn in der Nacht zum 14. Dezember 1990 erlag F. D. einem Herzinfarkt. Der Essay wurde posthum unredigiert in dem Band «Gedankenfuge» veröffentlicht. In den Gesprächen kurz vor seinem Tode wird neben den früher schon erwähnten Autoren St. W. Hawking und H. Weyl noch auf andere Autoren hingewiesen wie Teilhard de Chardin, Werner Heisenberg und Heinrich H. Keller mit seinem Aufsatz «Über die Rolle der Mathematik in der menschlichen Erkenntnis» ${ }^{13}$.

Diese Übersicht ist sicher nicht vollständig, doch gibt sie einen Einblick in die Werkstatt des Schriftstellers. Auf das bildernische Werk konnte aus Platzgründen nicht eingegangen werden.

9 Wittenberg, A. I.: Vom Denken in Begriffen. Mathematik als Experiment des reinen Denkens. Basel/Stuttgart: Birkhäuser Verlag, 1968.

10 Bd. 7, S. 710.

11 Bd. 7, S. 758-763.

12 Bd. 7, S. 733ff.

13 NZZ, 1988. 


\section{Gefährliche Entwicklungen}

In der Komödie, oder besser Tragikomödie «Die Physiker» nimmt Dürrenmatt kritisch Stellung zu den Folgen der modernen naturwissenschaftlichen Erkenntnisse und der daraus abgeleiteten Technik ${ }^{14}$. Möbius hat das Problem der Gravitation gelöst und die einheitliche Feldtheorie gefunden, die lange gesuchte Weltformel, die Quantenmechanik und Relativitätstheorie verbindet. Er kennt und fürchtet die daraus ableitbaren Konsequenzen, wenn dieses Wissen in falsche Hände gerät:

«Neue unvorstellbare Energien würden freigesetzt und eine Technik ermöglicht, die jeder Phantasie spottet, falls die Untersuchung in die Hände der Menschen fiele.»

Deshalb spielt Möbius den Verrückten und zieht sich in eine Nervenheilanstalt zurück unter Verzicht auf ein eigenes Leben. Möbius:

«Unsere Wissenschaft ist schrecklich geworden, unsere Forschung gefährlich, unsere Erkenntnis tödlich. Es gibt für uns Physiker nur noch die Kapitulation vor der Wirklichkeit. Wir müssen unser Wissen zurücknehmen, und ich habe es zurückgenommen. Es gibt keine andere Lösung ...» ${ }^{15}$.

Die Tragik ist, dass die einzige wirklich Verrückte in dem Ensemble, die Anstaltsärztin, seine Unterlagen, bevor er sie verbrennt, bereits photokopiert hat und in grossem Stile, machtbesessen, industriell ausschlachtet. Es geht dem Autor um die Abwägung und Bewertung der beiden Positionen Freiheit der Forschung und Verantwortung des Forschers - darf man alles tun, was man kann? -, die in einem gewissen Gegensatz zueinander stehen. Dürrenmatt löst die Frage, indem er die «schlimmstmögliche Wendung» wählt: Die Weltformel in der Hand einer Irren. Und schliesslich resignierend:

«Was einmal gedacht wurde, kann nicht mehr zurückgenommen werden.» ${ }^{16}$

Ein apokalyptisches Szenario führt uns der Autor auch in der Laudatio auf Michail Gorbatschow vor Augen, 1990 ${ }^{17}$. Zunächst bringt er eine kurze und präzise Beschreibung der Voraussetzungen für die Entstehung und erste Anwendung der Atombombe - von Otto Hahn bis Hiroshima. Dann die Weiterentwicklung zur Wasserstoffbombe und ihre Verbreitung. Die psychologische Auswirkung:

«Wie die Explosion einer Atombombe durch die Kernkettenreaktion einer kritischen Menge des Uranisotops 235 entsteht, löste die Atombombe, als wäre sie selber die kritische Menge an Furcht, eine Kettenreaktion an Furcht aus. Es war eine Furcht besonderer Art. Seit jeher wohnt dem Menschen die Furcht inne, mit der Atombombe lernte der Mensch sich selber

14 Bd.2, S. 191.

15 Bd. 2, S. 196.

16 Bd. 2, S. 207.

17 Bd. 7, S. 917-919. 
fürchten, es wurde seine eigene Apokalypse. Der Mensch war fähig geworden, die Menschheit zu zerstören. Er hatte gelernt, die Kraft einzusetzen, welche die Atomkerne zusammenhält, die Kraft, die entfesselt wird, wenn sich Materie in Energie verwandelt.»

In dem Essay «Die vier Verführungen des Menschen durch den Himmel» ${ }^{18}$ wird der Mondflug kritisch unter die Lupe genommen.

«Es ist leichter, auf den Mond zu fliegen, als mit anderen Rassen friedlich zusammenzuleben, leichter, als eine wirkliche Demokratie und einen wirklichen Sozialismus durchzuführen...»-

«Bloss, weil uns der Himmel verführte, ihn zu erobern, statt das, was uns allein gehört, vernünftig zu gestalten: unsere Erde. Es gibt keine andere Heimat, und jeder Fluchtversuch ist eine Utopie. Der Weltraumflug hat nur dann einen Sinn, wenn wir durch ihn die Erde entdecken und damit uns selber. Am 20. Juli 1969 bin ich wieder ein Ptolemäer geworden.»

Die seither vergangenen 25 Jahre haben die Richtigkeit der Kritik belegt.

Im «Porträt eines Planeten» ${ }^{19}$ lässt F. D. einen Atomwissenschaftler (Henoch) sagen:

«Ich erforschte das Innere des Atoms - Ich untersuchte die Beschaffenheit der Sonne - und versuchte die Struktur des Alls zu erfassen. - Ich fütterte mit meinen Kenntnissen die Computer. Die Computer gaben Formeln von sich, die ich gerade noch begriff ... Ich fütterte die Computer wieder, die eine Formel von sich gaben, die nur noch die Computer begriffen, ohne sie zu verstehen. Jetzt starre ich immer wieder auf die Formel, die das letzte Geheimnis dieser Welt bedeutet, ... die einen Sinn hat, den ich nicht verstehe, auf die sinnlose Formel der Welt.»

Diese kritische Zukunftsvision, von der wir vielleicht gar nicht mehr so weit entfernt sind, wird in dem Gedicht «Elektronische Hirne» erneut aufgegriffen und fortgeführt: Computer, die sich von Generation zu Generation weiterentwickeln, verselbständigen und über den Menschen hinauswachsen:

«Bis sie endlich Gott erkennen, ohne ihn zu verstehen ... Gefallene Engel.» ${ }^{20}$

In einer grotesken satirischen Science-Fiction-Erzählung spinnt F. D. diesen Gedanken weiter. Es wird von einem Computerzeitalter 10000 Jahre nach unserer Zeit berichtet, in dem «die Wirklichkeit die Summe aller ständig anwachsenden, im Zentralcomputer gespeicherten Fakten is $t \gg^{21}$. In dieser Story wurde der Mensch von Computern gezüchtet - der Gedanke, dass es auch umgekehrt gewesen sein könnte, wird empört als absurd zurückgewiesen -, um sein Hirn zu studieren. Doch wurde der Versuch bald aufgegeben, da die Computer sehr viel weiter entwickelt waren, so dass man der Menschheit nicht mehr bedurfte. Auch dieser Essay aus dem Jahre 1989 weist viele Parallelen zu den Ergebnissen der KL-Konferenz von Los Alamos aus dem Jahre

18 Bd. 7, S. 611-615, 1969.

19 Bd. 2, S. 783/784, 1970.

20 Bd. 7, S. 608.

21 «Der Versuch», 1989, in: Gedankenfuge, S. 106-131. 
1987 auf, die das evolutionäre Entstehen einer neuen Klasse von Computer«Organismen» prophezeit. In «Wissenschaft und Anarchismus» wird die o.g. Vision noch einen Schritt vorangetrieben ${ }^{22}$.

«... dass diese grausame Welt nur noch durch einen Computer bewältigt werden kann - durch eine aussermenschliche, nicht mehr emotionale Vernunft, durch die reine Vernunft eben, die alles, was nicht vernünftig ist, eliminiert, und sei es, dass auf blosses Übergewicht hin die automatisch durchgeführte Hinrichtung erfolgt ... Es könnte ja auch sein, dass diesem Computer, der das Weltall als Ganzes überblickt, das Leben vernünftigerweise nicht einleuchtet ...»

und in «Achterloo» ${ }^{23}$ schreibt er: Die Evolution hat unser Menschenhirn befähigt, Prothesen zu schaffen, auch Hirnprothesen, Computer.

«Der von seinem Schöpfer, dem Menschen befreite Computer ist der Sinn des Menschen, in ihm findet der Mensch seine Vollendung.»

Und weiter lässt er seinen Protagonisten sagen: Die alte Menschheit, überflüssig geworden, löst sich in einem Feuerbad auf, aus dem die neue Menschheit, jene der künstlichen Gehirne hervorgeht. Der Mensch «in einem Irrkreis der Evolution».

Kurz vor seinem Tode fasste F. D. in einer apokalyptischen Vision noch einmal seine Sorge um die Zukunft der Menschheit zusammen anlässlich eines Gespräches mit Sven Michaelsen ${ }^{24}$ :

«Die Weltbevölkerung explodiert wie das Weltall, worin wir leben. Uns droht eine Klimakatastrophe. Das zeigt doch, dass unser jetziger Friede die fatale Eigenschaft hat, den Krieg zu integrieren.» - «Wir haben uns eine Katastrophenwelt gebaut. Ein zerstreuter Laborant führt die Explosion einer Atombombenfabrik herbei, ein schläfriger Programmierer fabriziert eine Fehlschaltung in den Pentagon-Computern, einem unachtsamen Gentechniker entwischen seine Virus-Kulturen - in diese Welt der apokalyptischen Pannen führt unser Weg. Deshalb muss die Literatur fragen, ob die Menschheit nicht in einer evolutionären Krise steckt und auf ihr Ende zugeht.»

Das Fazit, das F. D. aus dieser prekären Situation zieht, lautet:

«Es gibt Risiken, die man nie eingehen darf: Der Untergang der Menschheit ist ein solches.» ${ }^{25}$

\section{Physik}

Physikalische Gedankengänge finden sich verstreut über das ganze Oeuvre, besonders aber im Nachlassband «Gedankenfuge», in den «Stoffen I-III» und «IV-IX» und in den Essays.

22 Bd. 7, S. 216.

23 Bd. 3, S. 400 und 401

24 Über die Grenzen, S. 17 und 19.

25 Die Physiker, Bd. 2, S. 195. 
In der Geschichte «Der Winterkrieg in Tibet ${ }^{26}$ lässt der Autor seinen Helden im Höhlenlabyrinth tief im Inneren des Himalajagebirges über die Analogie zwischen den Gesetzen der Thermodynamik, die in der Loschmidtschen Konstante das Verhältnis von Masse, Volumen, Temperatur und Druck eines idealen Gases ausdrücken, und den Gesetzen der menschlichen Institutionen nachdenken. Dabei kommen ganz ähnliche Beziehungen zum Vorschein, wie sie sich an den Atomen in einem Stern abspielen oder wie sie auf den folgenden Seiten mit einer kosmologischen Metapher beschrieben werden (s. Kapitel Kosmologie). In den «Überlegungen zum Gesetz der grossen Zahl ein Versuch über die Zukunft» ${ }^{27}$ wird der Gedanke erneut aufgegriffen:

«Wie in der Thermodynamik gewisse Gesetze erst auftreten, wenn «sehr viele> Moleküle beteiligt sind (Loschmidtsche Konstante: $6.02 \times 10^{23}$ ) - während die Bewegungen der einzelnen Moleküle dem Zufall unterworfen sind -, so werden gewisse Gesetze erst bei «sehr vielen> Menschen wirksam (Erdbevölkerung 4 Milliarden), etwa jenes des Primats der Gerechtigkeit vor der Freiheit.» «Dass ich so viel vom Zufall rede, ist nicht ein dramaturgischer Trick, ... ich trage nur dem Gesetz der grossen Zahl Rechnung. $»^{28}$

In dem Essay «Die Brücke» ${ }^{29}$ geht es um die absolute Wahrheit, der man sich nur asymptotisch nähern kann, ohne sie nach Dürrenmatts Meinung jemals zu erreichen:

«... ihre (viele Physiker) physikalische Weltformel wäre dann auch eine metaphysische.» ${ }^{30}$

Hier spielt der Autor auf die Bemühungen der Physiker, vor allem Heisenbergs, an, eine Weltformel zu finden, die die Relativitätstheorie mit der Quantentheorie verbindet. Auch im weiteren Verlauf des Essays schimmert wieder das Gedankengut der Quantenmechanik durch: Unschärferelation, das Konzept der 'Wahrscheinlichkeitswelle〉(Bohr, Kramers, Slater), das von Max Born als ein Mass für die Wahrscheinlichkeit eines Ereignisses interpretiert wird; bei Dürrenmatt stehen dafür Begriffe wie Möglichkeit, Wahrscheinlichkeit, Unwahrscheinlichkeit, logische Folgerichtigkeit, kausaler Zusammenhang und Zufall des Eintritts eines bestimmten Ereignisses.

«So bleibt mir $^{31}$ denn nichts anderes übrig, statt der Wahrheit ... Möglichkeiten wiederzugeben.»

26 Bd. 6, S. 99, Stoffe I-III.

27 Bd. 7, S. 691.

28 Bd. 7, S. 693.

29 Bd. 6, S. 394 bis 419, Stoffe IV-IX.

30 Bd. 6, S. 396.

31 Bd. 6, S. 404. 
Daraus leiten sich die von ihm häufig als Stilmittel benutzten Fiktionen ab: anstelle des Denkens <über die Welt > das 〈Denken von Welten ${ }^{32,33}$ :

«Zielt die Dramatik auf eine ‘Wiedergabe der Welt», muss sie sich daher dem Satze Newtons unterwerfen: Hypotheses non fingo. Sie wird «naturwissenschaftlich〉 abhängig von der Theorie über die Welt, auf die sie sich stützt ...»

\section{Er fährt fort ${ }^{34}$ :}

«Eine physikalische Fiktion scheint mir eine bewusste Denktechnik zu sein, mit der ich der Wirklichkeit gleichsam eine Falle stelle und ihr so eine Antwort entlocke, die ich jedoch nur physikalisch zu deuten vermag; künstlerische Fiktionen scheinen mir Denktechniken zu sein, die darauf ausgehen, scheinbare Wirklichkeiten zu errichten. Wird durch eine physikalische Fiktion die Wirklichkeit befragt, wird die Wirklichkeit durch die künstlerische Fiktion künstlich hergestellt.» ${ }^{35}$

Auch hier wieder eine typische Verquickung naturwissenschaftlich-mathematischen Denkens und künstlerischer Kreativität. Doch verfolgen wir den oben begonnenen Gedankengang aus dem Essay «Die Brücke» weiter. $\mathrm{Zu}$ den aufgeführten Begriffen kommt nun auch noch der Begriff des Glaubens hinzu ${ }^{36}$ :

«... ohne Glauben ist auch das Vermuten nicht möglich, das immer ein Überschreiten der Brücke zwischen Gesichertem und Ungesichertem ist; ... ohne Glauben, es könne so sein, wie ich es mir vorstelle, ... ist keine Wissenschaft möglich.» ${ }^{37}$

Das Fortschreiten des Erkenntnisprozesses, von der modernen Physik im ersten Drittel des 20. Jahrhunderts induziert, wird von Dürrenmatt in diesem Essay aufgenommen und literarisch und philosophisch verarbeitet. So schreibt er ${ }^{38}$ :

«Nur im rein Logischen gibt es absolut Sicheres wie etwa den Lehrsatz des Pythagoras. - Alles Zukünftige ist bloss wahrscheinlich, wobei im Wahrscheinlichen auch das Unwahrscheinliche eingeschlossen ist: Das Wahrscheinliche liegt noch in unserer ungefähren Berechenbarkeit ... das Unwahrscheinliche dagegen, das ebenfalls einzutreten vermag, aber nicht dazu genötigt ist, liegt gänzlich ausserhalb des Vorausberechenbaren.»

32 Bd. 7, S. 70, Essays und Gedichte, Theater.

33 Bd. 7, S. 71.

34 Bd. 7, S. 152.

35 Bd. 7, S. 70 und 152.

36 Bd. 7, S. 415.

37 Anmerkung: Wie könnte man besser die Bedeutung der Intuition und Imagination für die naturwissenschaftliche Erkenntnis umschreiben: Es genügt eben nicht, empirische Daten logisch zu verknüpfen; vielmehr bedarf es der schöpferischen Imagination des Wissenschaftlers. Mann kann diesen Akt als Hypothesenbildung, Theorie oder Glauben bezeichnen, wie Max Planck es 1922 ausgedrückt hat: «Auch in der Physik gilt der Satz, dass man nicht selig werden kann ohne den Glauben.» Zu ganz ähnlichen Vorstellungen gelangte Einstein im Alter. Auch Wolfgang Pauli schreibt dem schöpferischen, geistigen Prozess einen hohen Stellenwert zu, reine Logik und das Aneinanderreihen empirischer Daten genügen nicht.

38 Bd. 7, S. 417. 


\section{Daraus folgert er,}

«dass die Wirklichkeit eine Unwahrscheinlichkeit darstellt, die eingetreten ist. - Der Satz ist kosmologisch und behauptet, dass die Welt unwahrscheinlicherweise so ist, wie sie ist, was nicht dagegen spricht, dass sie so ist, wie sie ist.»

Dieses Spiel von Zufall und Notwendigkeit konkretisiert er an einem fiktiven Ereignis: Ein Student, F. D., wird beim Überqueren einer Brücke von einem Meteor erschlagen. Die Bahn des Meteors wird als absolut gesetzmässig, notwendig, beschrieben. Das Verhalten des Studenten hingegen wird mannigfach variiert und ergibt erneut das Bild des Zufalles, des nicht Vorherbestimmbaren. In dieser Parabel beschreibt F. D., ohne das Wort stochastisch zu benutzen, einen stochastischen Prozess, das zufallsabhängige und deshalb nur statistisch zu erfassende Verhalten der individuellen Träger eines dynamischen Prozesses.

In dem Essay «Abhängigkeit der Wissenschaft» berichtet F. D. von einem Besuch im CERN 1974, verarbeitet und niedergeschrieben $1976^{39}$. Er beschreibt die Superprotonensynchrotrone und die daran durchgeführten Untersuchungen an den Bausteinen des Atomkerns. Dabei stellt sich ihm die Frage:

«... ob der Mensch nicht Gefahr laufe, schliesslich Ur-Teilchen zu erfinden statt zu finden.»

Diese Frage ist nur zu berechtigt, hat doch die Chemie in Zusammenarbeit mit der Physik auch Atome geschaffen, die Trans-Urane, die in der Natur nicht vorkommen. Auch die Geschichte des Neutrinos, kleinster Teilchen ohne Ladung und fast ohne Masse, wie F. D. sehr richtig bemerkt, beginnt mit ihrer «Erfindung) durch Wolfgang Pauli 1931. Beim radioaktiven Beta-Zerfall ging immer ein wenig Energie verloren. Das durfte nach dem Satz von der Erhaltung der Energie und des Drehimpulses, einer der Grundlagen der Physik, nicht sein. So postulierte Pauli kleinste Teilchen, die mit Lichtgeschwindigkeit den Raum durcheilen und jedwede Materie durchdringen, als wäre sie ein leerer Raum, ohne Schaden anzurichten. Die von Dürrenmatt angegebene Zahl der Neutrinos, die pro Sekunde unserern Körper durchfliessen und von ihm mit $10^{14}$ beziffert werden, muss nach heutiger Kenntnis (1992) auf $10^{11}$ korrigiert werden.

Erst 1956 wurden die lange Zeit als Hirngespinst verschrieenen Neutrinos gefunden, und seit 1990 gibt es exaktere Daten durch das europäische Gallex-Experiment in den Abruzzen. Wenn Dürrenmatt von der «Doppelnatur» der Atomkern-Teilchen spricht, so entspricht das der Tatsache, dass Masse 
und Energie ineinander übergehen können $\left(\mathrm{e}=\mathrm{mc}^{2}\right)$ und dass diese kleinsten Teilchen bei geschickter Versuchsanordnung sowohl als korpuskuläre Elemente als auch als elektromagnetische Welle auftreten können. Beispiele hierfür sind die Paarbildung, d.h. die Umwandlung eines Photons (elektromagnetische Welle) in ein Elektron-Positron-Paar und somit Umwandlung von Energie in Materie. Das Komplementäre ist die Annihilation, die Paarvernichtung unter Aussendung von Strahlen, Umwandlung von Materie in Energie. Die Frage Dürrenmatts,

«was denn ein Neutrino eigentlich sei, was man denn unter einem masselosen Teilchen eigentlich verstehe, das um sich selbst rotiere ...» ${ }^{40}$

beantwortet der CERN-Physiker:

«Niemand sei sich klar darüber und könne sich klar darüber sein, was denn eigentlich, ausserhalb der physikalischen Fragestellung, 〈in Wirklichkeit> diese Teilchen seien ...» «Dieses falle vielmehr in das Gebiet der philosophischen Spekulation und sei für die Physik irrelevant. $\rangle^{41}$

In dem grossartigen Essay Kunst und Naturwissenschaft ${ }^{42}$ geht es um die Denkweise des Naturwissenschaftlers. F. D. beschreibt hier eine der Nahtstellen zwischen Naturwissenschaften und Philosophie:

«Dem Naturwissenschaftler geht es in seinen Experimenten um die Naturgesetze. Er stellt fest, was sich in einem bestimmten Augenblick unter bestimmten Bedingungen ereignen muss. Ein wissenschaftliches Experiment beweist etwas.» ${ }^{43}$ : «Die Physiker denken sich eine Welt zurecht, in der die Naturgesetze zum Vorschein kommen ... Die Naturgesetze ... sind nicht seiend, sondern weisen auf Seiendes hin. Darum sind sie auch verifizier- und falsifizierbar, während die Ideen Platons weder widerlegbar noch beweisbar sind ... - Eine physikalische Idee muss fallengelassen werden, erweist sie sich als unhaltbar.»

Als Beispiel wird die Widerlegung des «Äthers durch die Experimente Michelsons und Morleys 1881 angeführt.

Dass einmal Gedachtes und Niedergeschriebenes nie ganz verloren geht, auch wenn es vorübergehend vergessen wird, zeigt F. D. im gleichen Essay, in dem $\mathrm{er}^{44}$ die kosmologischen und physikalischen Vorstellungen von Xenophanes über Parmenides, Platon, Aristoteles, Galilei, Newton, Kant bis hin zur moderen Urknall- und Atom-Theorie darstellt. Er vergleicht die Atomvision der Vorsokratiker mit der Atomidee Platons und dessen «märchenhafter Vorahnung der Moleküle» und beschreibt die Problematik der Annahme des leeren Raumes, des Nichts. An anderer Stelle ${ }^{45}$ ist zu lesen:

40 Bd. 7, S. 179.

41 Bd. 7, S. 180.

42 Bd. 7, S. 493.

43 Bd. 7, S. 552.

44 Bd. 7, S. 542-545.

45 Bd. 7, S. 482, Varlin. 
«Der leere Raum und das Nichts sind logische Konstruktionen, nicht Wirklichkeiten, so unfassbar uns die Realität erscheint, so ist sie doch existent wie das Leben. Dass wir fähig sind einen leeren Raum zu denken, der doch als Raum nur möglich ist, weil in ihm Dinge sind, Sterne, Menschen und Atome, oder wenn wir von einem Nichts reden, das von jedem Stein widerlegt wird, so lassen wir uns von der Sprache blenden.»

Damit gibt er getreu die aristotelische Vorstellung wieder, dass Raum Ortsbestimmung und Begrenzung von Körpern ist; es gibt keinen leeren Raum. Doch die heutige Begründung, wie sie z.B. St.W. Hawking gibt, lautet anders. Was wir uns als leeren Raum vorstellen, kann nicht völlig leer sein, weil dann alle Felder, das elektromagnetische und das Gravitationsfeld exakt gleich Null sein müssten. Der «leere» Raum ist angefüllt mit Feldern hoher Dichte.

Gross ist die Faszination, die Einsteins Formel $\mathrm{e}=\mathrm{mc}^{2}$ auf F. D. ausübt, jene Formel, welche die Überführbarkeit von Masse (m) in Energie (e) und umgekehrt über die Lichtgeschwindigkeit (c) beschreibt.

«... welch ein Denken ist dahinter verborgen, nicht nur das Denken eines Einzelnen, mehr noch das Denken vieler Jahrhunderte ...»46

Für das Verständnis der Prozesse im Inneren der Sterne, ja der Entstehung des Kosmos ist diese Formel ebenso unverzichtbar wie für die verhängnisvolle Entwicklung der Atom- und H-Bombe, die ohne sie nicht denkbar wäre. Das hat F. D. immer wieder betont. Aber auch in einem ganz anderen $\mathrm{Zu}$ sammenhang kommt er auf Einsteins Formel zurück: In dem Vortrag «Einführung Yasushi Inoue ${ }^{47}$ vergleicht er sie mit chinesischen Schriftzeichen.

«Nun hat sich die chinesische Schrift aus dem Bild entwickelt, die Formel aus einem langen Denkvorgang ... Stellt das chinesische Zeichen für Herbst ... gleichsam das abstrakte Bild dieser Jahreszeit dar, so bedeutet die Formel $\mathrm{e}=\mathrm{mc}^{2}$ die abstrakte Einsicht in das Wesen von Energie und Materie, auch hier, das chinesische Schriftzeichen bedeutet eine Metapher, die Formel ein Gleichnis ... Die Formel zielt nach der Ursache, das Zeichen nach dem Sinn ...»

Die beiden Gedankenstränge, weiterverfolgt, führen zu einem Vergleich der abendländischen mit der fernöstlichen Kultur.

In den nachgelassenen Essays «Gedankenfuge» $»^{48}$ und «Kabbala der Physik $»^{49}$ wird eine Diskussion (1988) zwischen dem Autor und seinem Freund Marc Eichelberg über die Einstein-Formel beschrieben. F. D. stösst sich an $\mathrm{c}^{2}$, dem Quadrat der Lichtgeschwindigkeit, und man stellt sich die Frage, warum erst jetzt, 9 Jahre nach dem Einstein-Vortrag in Zürich. Mathematisch stellt die Gleichung $\mathrm{e}=\mathrm{mc}^{2}$ zunächst den Identitätssatz $\mathrm{A}=\mathrm{A}$ dar. Doch wenn

46 Bd. 7, S. 753, Albert Einstein.

47 Bd. 7, S. 576 und 577.

48 Gedankenfuge, S. 49/50.

49 Ebenda, S. 132/133. 
man jetzt Energie, Masse und Lichtgeschwindigkeit einsetzt, bekommt man eine physikalische Aussage, nämlich, unter welchen Bedingungen Masse in Energie und umgekehrt umgewandelt wird. Den Konflikt der Physik sieht F. D. nun darin, dass in dieser Formel mit dem Quadrat der Lichtgeschwindigkeit gearbeitet wird, obwohl die Physik keine Geschwindigkeit kennt, die grösser ist als die Lichtgeschwindigkeit.

«Energie hat nur in ihrer Verbindung mit Masse und Zeit (?) einen Sinn. Die Zauberformel $\mathrm{e}=\mathrm{mc}^{2}$ sagt aus, wieviel Energie es braucht, die Masse in Nichts zu verwandeln, und da diese Umstände unmöglich sind, weil es die Geschwindigkeit von 90 Milliarden Kilometern in der Sekunde nicht gibt, bedeutet es, dass es diese Formel nur als Idee gibt, aber vielleicht auch, dass die reine Umwandlung von Masse in Energie nicht möglich ist, dass, was wir Energie nennen, Masse ist, nur in anderer Form, zerstrahlt in Partikel aufgelöst ... Nun ist das eine kabbalistische Auslegung einer physikalischen Formel.»

Diese Auslegung hält einer sachlichen Überprüfung nicht stand. Die Formel $\mathrm{e} \rightleftharpoons \mathrm{mc}^{2}$ bedeutet, dass sich Energie in Masse und umgekehrt verwandeln kann und nicht in Nichts, was F. D. weiter oben selber sagt. Man spürt in diesen Sätzen das Ringen des Autors um die Verbalisierung des Unvorstellbaren, des Unanschaulichen. Marc Eichelberg setzt dagegen, dass

«... Masse und Energie äquivalente Erscheinungsformen desselben Phänomens seien. $\mathrm{c}^{2}$ sei rein mathematisch nichts als ein Multiplikand.»

F. D. bleibt nach dieser Diskussion unzufrieden zurück und kommentiert die Erklärung seines Freundes mit den vielsagenden Worten:

«Die Physik spielt erkenntnistheoretisch mit gezinkten Karten.»

Und einige Seiten später ${ }^{50}$ - man merkt, wie ihn das Problem nicht loslässt -:

«Kabbalistisch verstanden, stellt $\mathrm{e}=\mathrm{mc}^{2}$ eine magische Formel dar: die physikalische Unmöglichkeit des Begriffs $\left(\mathrm{c}^{2}\right)$... weist darauf hin, dass die Umwandlung von Materie in reine, das heisst potentielle Energie, oder umgekehrt, ... nur in der Idee oder im Nullpunkt von Raum und Zeit möglich ist. $\mathrm{e}=\mathrm{mc}^{2}$ ist kabbalistisch die Formel des Urknalls.»

Noch eine andere Widersprüchlichkeit fällt in dem Essay «Kabbala der Physik» ins Auge ${ }^{51}$ :

«Elektron und Positron, Materie und Antimaterie annihilieren sich gegenseitig, sie verschwinden aus dem Universum und lassen einen Energiestoss zurück: Licht.»

So weit, so gut. Nun aber folgt ein Gedanke, dem man nicht zustimmen kann:

«Die Antimaterie wurde mathematisch vorausgesagt, indem ein Minuszeichen als negative Materie interpretiert wurde, derart, dass auf jedes mögliche Partikel ein Antipartikel käme, was eine vollständige Durchdringung unseres Universums durch ein Antiuniversum bedeuten würde ...»

50 Ebenda, S. 135/136.

51 Ebenda, S. 134/135. 
Das aber ist nicht möglich, denn in Gegenwart von normaler Materie erfolgt völlige Zerstrahlung durch Paarvernichtung (Annihilation). In einem von Normalmaterie freien Raum ist Antimaterie genauso stabil wie Materie, so dass man sich auch ein Universum aus Antimaterie vorstellen kann. Damit ist auch die Deutung des Hexagramms, aus zwei gleichen Dreiecken konstruiert, als Symbol für zwei Welten, eine unsichtbare (Antimaterie) und eine sichtbare (normale Materie), die sich gegenseitig durchdringen, ins Reich der Kabbala, nicht jedoch der Physik zu verweisen.

Es kann nicht oft und eindringlich genug darauf hingewiesen werden, dass die Essays im Nachlassband «Gedankenfuge», besonders aber der Essay «Kabbala der Physik», unredigiert posthum veröffentlicht wurden, was die Widersprüche und Ungenauigkeiten erklärt. Wer Dürrenmatts Arbeitsweise kennt, der weiss, dass jeder Text immer wieder überarbeitet, verbessert und oftmals sogar neu geschrieben wurde, ehe F. D. das Placet für die Veröffentlichung gab. Von Kabbala der Physik war der Überarbeitungs- und Korrekturtermin schon festgelegt, sein sachverständiger Freund, Marc Eichelberg, in Neuchâtel eingetroffen, als der plötzliche Tod dem Autor die Korrekturfeder aus der Hand nahm.

Auch der Begriff des Schwarzschildradius wird nicht korrekt interpretiert:

«Der Schwarzschildradius ist das sich immer wieder ausdehnende und zusammenfallende Weltall.» ${ }^{52}$ oder

«... das Ende der Materie und der Anfang der Antimaterie sind der Schwarzschildradius ...»

Vielmehr ist der Schwarzschildradius definiert als der Radius, bei dessen Erreichen eine Masse zum Schwarzen Loch wird. Der Radius beträgt für eine Sonnenmasse 2,95 $\mathrm{km}^{53}$. Oder die St. W. Hawkingsche Definition:

«Der Schwarzschildradius ist der Abstand der Wellenfront im Ereignishorizont vom Ursprung der Wellen. $»^{54}$

Ähnlich mystifiziert wird die Möbius-Fläche. Sie wird zwar richtig als eine um $180 \mathrm{Grad}$ in sich selbst verdrehte, zweidimensionale Fläche beschrieben, als Prototyp einer nicht orientierbaren Fläche, bei der man von einer Seite auf die andere gelangen kann, ohne den Rand zu überschreiten. Man kann F.D. auch noch folgen, wenn er die Möbius-Fläche als Metapher dazu benutzt, den Übergang von Antimaterie zu Materie - vice versa - in der Singularität des Urknalls oder des Schwarzen Loches darzustellen. Damit greift F. D. auf neue Hypothesen zurück, die einen pulsierenden Kosmos beschreiben, oder wie St. W. Hawking, die Bildung immer neuer Tochter-Universen.

52 Ebenda, S. 138.

53 Störig, H. V.: Knaurs Moderne Astronomie. München: Droemer Knaur, 1992, S. 182.

54 Hawking, St. W.: Einsteins Traum. Reinbek: Rowohlt, 1993, S. 100. 
Dieses Kapitel abschliessend, sollte noch eine groteske Science-FictionGeschichte ${ }^{55}$ erwähnt werden. Hier wird das Problem aufgegriffen,

«Dass die Wirklichkeit, die dokumentiert werden soll, beobachtet werden ... muss, ... (und) dass dieses Beobachten des Beobachteten das Beobachtete zu verfremden, ja, zu verändern vermag.»

Wieder dringt naturwissenschaftliches Gedankengut (Unschärferelation aus der Quantenmechanik von Werner Heisenberg: der Messvorgang im subatomaren Bereich beeinflusst die Konstellation der kleinsten Teilchen so sehr, dass nur statistische Aussagen über ihre räumliche Anordnung zu einem bestimmten Zeitpunkt gemacht werden können) in seine Betrachtungsweise ein und bestimmt sein Denkschema:

«Das Wissen, beobachtet zu werden, ist deshalb das eigentliche Problem.»

Analog: der Messvorgang ist das eigentliche Problem. Daraus folgt:

«die unmittelbare Wirklichkeit ist nicht dokumentierbar.» ${ }^{56}$

Anlass zu diesem Gedankengang war eine Alltagsbegebenheit. Wenn Dürrenmatt auf der Terrasse seines Neuchâteler Hauses sass, wurde er häufig von Spaziergängern von einem Weg oberhalb seines Grundstückes beobachtet. Deren Verhalten änderte sich schlagartig, wenn er nun seinerseits zum Fernglas griff und die Beobachter beobachtete. Es gehört die Einbildungskraft und Logik eines Dürrenmatt dazu, aus dieser simplen Begebenheit eine tiefsinnige analytische Geschichte zu machen.

\section{Kosmologie}

Kernpunkt seiner kosmologischen Betrachtungen ist die Urknall-Hypothese, der wir immer wieder im Dürrenmattschen Oeuvre begegnen. Schon der erste Satz des Essays «Das Hirn $»^{57}$ enthält eine konzentrierte Fassung dieser Hypothese:

«Wenn die moderne Kosmologie sich vorstellt, die Welt sei aus dem Nichts entstanden, als Explosion eines dimensionslosen Punktes, worin nicht nur alle Materie und Energie des Weltalls, sondern auch dessen Zeit und Raum zusammengezwängt waren, eine nur mathematisch mögliche Konstruktion, so können wir uns auch statt dieses rein hypothetischen Punktes ein reines Hirn vorstellen.»

55 Gedankenfuge, S. 109/115.

56 Ebenda, S. 132-140.

57 Bd. 6, S. 539. 
Des weiteren beschreibt er die interstellare Materie, die Gasnebel, Sonnen, Planeten, Quasare, stabile, verpuffende, unendlich sich ausdehnende oder kollabierende Weltalle, Weltalle aus Antimaterie ${ }^{58}$. Im «Winterkrieg in Tibet», 1981, Neufassung $1990^{59}$ wird nun Schritt für Schritt die Geschichte eines Sternes, eines Sonnensystems entwickelt bis hin zum Zerfall: Eine Ursonne, ein Protostern, wird aus dem Gasnebel herauskondensiert. Seine Zusammensetzung aus Wasserstoff, Kohlenstoff, Sauerstoff, Neon, Helium und den aus Supernova-Explosionen hereingeschossenen schweren Elementen wird detailliert analysiert, ebenso die Einwirkung des Drehimpulses, der Gravitation und Temperatur. Die Entwicklung zum Roten Überriesen, zum Gelben Zwerg, die Entstehung der Planeten, die Druck-, Temperatur- und Energie-Verhältnisse im Sonneninneren werden subtil beschrieben. Weiter geht der Weg zum blauen Stern und schliesslich zur Nova, die zu einem Weissen Zwerg und zuletzt zu einem schwarzen Zwerg schrumpft. Aber auch die alternative Entwicklung von Sternen, die das 1,44fache unserer Sonnenmasse überschreiten - die berühmte Grenze Chandrasekhars wird aufgezeigt: Über die Super-Nova-Explosion zum Neutronenstern mit seiner unvorstellbaren Dichte oder gar zum Schwarzen Loch. Und nun folgt ein für F. D. typischer Kunstgriff: Er vergleicht die Geschichte der Sterne mit der Menschheitsgeschichte.

«Ist die Sonne eine Ansammlung von Wasserstoff, ist der Staat eine Ansammlung von Menschen. Beide sind den gleichen Gesetzen unterworfen. Beide sind stabil, wenn ein Druck-, Energie- und Oberflächengleichgewicht besteht. Bei beiden wirkt die Gravitation. Sie beginnt einen Kern und um ihn herum eine Konvektionszone zu bilden ... Auf den Staat bezogen: Die Konvektionszone stellt die Behörden, der Kern das Volk dar ... Das Druckgleichgewicht eines stabilen Staates besteht darin, dass sich der Einzelne möglichst frei bewegt, so frei nämlich, wie es den anderen Einzelnen gegenüber möglich ist; je grösser deren Masse, desto beschränkter wird die Freiheit des Einzelnen: der Druck auf den Einzelnen ist angestiegen und damit die Temperatur innerhalb der Masse; sie beginnt den Staat zu spüren, Emotionen gegen den Staat werden frei usw.»

Eine zeitlang wird der steigende Innendruck von den Behörden (Konvektionszone) aufgefangen; aber immer häufiger durchbrechen politische, kriminelle und terroristische Katastrophen wie «Protuberanzenausbrüche» die Konvektionszone: es kommt zu einem neuen Weltkrieg mit Atom- und Wasserstoffbomben (Zustand einer Supernova).

«Nach der Supernova der Endzustand: die Menschheit als Neutronenstern. Der auf wenige bewohnbare ... Landstriche zusammengepferchte Rest der Menschen ... ist zu einer einzigen Konvektionszone geworden, astronomisch mit einem <entarteten Neutronengas zu vergleichen, zu einer total verwalteten Masse .... ${ }^{60}$

58 Bd. 6, S. 543, Das Hirn.

59 Bd. 6, S. 100.

60 Bd. 6, S. 107ff. Der Winterkrieg in Tibet. 
Hier scheint das naturwissenschaftliche Thema mehr als nur eine Metapher zu sein; vielmehr gewinnt man den Eindruck, dass der Autor mit der ihm eigenen naturwissenschaftlichen Denkweise an politische Themen analysierend herangeht. Ganz ähnlich sind die Gedankengänge in den Betrachtungen über den Mitmacher: «Bill und die Ohnmacht $»^{61}$. Auch hier wird der Staat mit der Sonne verglichen, die explodieren oder in sich zusammenstürzen kann. Es gibt keine stabile Gesellschaftsordnung ausser vielleicht der chaotischen.

In der Auseinandersetzung mit Rudolf Kassners «Zahl und Gesicht» ${ }^{62}$ stösst er auf dessen «groteske Fiktion» einer unendlichen Welt, die heute von der «physikalischen Kosmologie als paradoxe Theorie» aufgestellt wird:

«... so etwa, dass die Welt einen Anfang genommen habe, aus dem Urknall heraus ...»

Was Kassner 1918 imaginierte, ist heute z.T. experimentell belegt,

«etwa durch die Expansion des Weltalls oder die Hintergrundstrahlung.» «Gesicht und Zahl verschmelzen sich gegen seinen (Kassners) Willen in einer einzigen Kernfusion zum Begriff ...»

Selbst bei der Erwähnung des Herzsekundentodes seiner Frau dient die Kosmologie als Metapher:

«Ich kam von der Vorstellung nicht los, meine Frau würde sich mit Lichtgeschwindigkeit von mir entfernen, jede Sekunde 300000 Kilometer, 18 Millionen Kilometer jede Minute, mehr als 1000 Millionen jede Stunde ...»- «... der Tod ist nur von aussen darstellbar und stellt sich von aussen dar, sei es als ferne, ausgefranste nelkenähnliche Wolke, im Sternbild des Stiers, der Überrest einer Sonne ...» ${ }^{63}$

Bei der Betrachtung des Andromeda-Nebels kommen dem Autor Gedanken über die Relativität der Zeit und der Gleichzeitigkeit ${ }^{64}$

«...A Auch dieser liegt unerreichbar in der Vergangenheit, zweieinhalb Millionen Jahre zurück, sein Licht, das wir erblicken, verliess ihn im ersten Aufdämmern der Menschheit ... - unsere Milchstrasse, deren Mittelpunkt wiederum in der Vergangenheit liegt, in einer anderen freilich, dreissigtausend Jahre zurück, ...»- «Wir sind nicht von Vergangenheit umgeben, sondern von Vergangenheiten, von einer Welt von über- und durcheinandergewobenen «Erinnerungsbildern>.»

In «Paul Flora ${ }^{65}$ greift er diesen Gedanken erneut auf.

«Schliesslich treiben wir in einem Meer von Vergangenheit dahin, und blicken sie (die Sterne) auf uns, glotzen ihnen Dinosaurier entgegen.»

61 Bd. 7, S. 227.

62 Bd. 6, S. 288 und 290.

63 Bd. 6, S. 329, Stoffe IV-IX, Begegnungen.

64 Bd. 6, S. 50ff. Stoffe I-III.

65 Bd. 7, S. 501. 
Noch grösser ist die Entfernung und damitVergangenheit ferner Galaxien und Quasare, Milliarden Lichtjahre, fast dem Alter des Weltalls entsprechend.

«Würden die Vergangenheiten aufgehoben, stürzte das Weltall vom Rande her auf uns zu. Der Urzustand der Welt stellt sich wieder her, von dem wir uns kein Bild machen können, weil er bildlos wäre, reine vergangenheitslose Gegenwart, in die sich die Zeit nicht mehr vorwärtsstürzen könnte, um wieder Vergangenheit zu erzeugen, und das nur, weil sie sich von der Zukunft her zurückgestürzt hätte: die Welt in die kleinstmögliche Zeiteinheit zurückgepresst, in ein Chronon, eine Zeitspanne im Bereich einer quadrillionstel Sekunde, mehr noch, die Welt als Zeit- und Raumlosigkeit, die Welt als mathematischer Punkt. So verhilft uns allein die Vergangenheit zu Bildern, das Auseinanderstieben der Milchstrassen, die Expansion des Weltalls ... ${ }^{66}$

Mit diesen dramatischen Worten und Bildern beschreibt F. D. eine der Möglichkeiten der zukünftigen Entwicklung des Kosmos, den Gravitationskollaps bis hin zum Zustand der Singularität, wie sie als Ausgangspunkt bei der Urknall-Hypothese angenommen wird. Was wir im Kosmos als Gegenwart erkennen, ist in Wirklichkeit schon Vergangenheit, denn die Information braucht Zeit, ehe sie uns erreicht, ihr Ursprung ist bereits «versunken im Abgrund des Nicht-mehr-Seins.» ${ }^{67}$

In seiner Schrift über «Jef Verheyen», $1982^{68}$, spricht F. D. über das schwarze Loch:

«Wir leben heute in einer Zeit, in der ein Loch an sich, ein Loch als Singularität, als sog. Schwarzes Loch, ... eine kosmische Konsequenz des wissenschaftlichen Denkens über die Gravitation darstellt, das vor 300 Jahren mit Newton begonnen und das Einstein weitergeführt hat.»

«Einsteins ... berühmte Gleichung, $\ldots \mathrm{E}=\mathrm{mc}^{2}$ ist einfach und in ihrer Einfachheit von bestechender Schönheit.... Sie versinnbildlicht ein Denken, das mehr als jedes andere Denken die Welt veränderte, kosmologisch, indem es nicht, wie Einstein hoffte, zum Schauen der praestabilierten Harmonie führte, sondern zur Vision einer praestabilierten Explosion, zu einem monströsen, auseinanderfegenden Weltall voller Supernova, Gravitationskollapse, Schwarzer Löcher, zu einem Universum der Weltuntergänge, das sich von Jahr zu Jahr unheimlicher, ja tückischer zeigt ...» ${ }^{69}$

In seinen Anmerkungen zu dem Vortrag «Albert Einstein $»^{70}$ verweist Dürrenmatt auf einen Artikel in der Neuen Zürcher Zeitung vom 24.9.73, in dem die von Collins und Hawking festgestellte Isotropie des Universums und das vermutete Vorhandensein vieler Tochteruniversen beschrieben wird. In der Laudatio auf Michail Gorbatschow ${ }^{71}$ mit dem Titel «Die Hoffnung, uns am eigenen Schopfe aus dem Untergang zu ziehen», beschreibt F. D. erneut die Kosmogonie und setzt sie in Analogie zur Politik:

66 Bd. 6, S. 51/52, Stoffe I-III.

67 Bd. 3, S. 348, Achterloo.

68 Bd. 7, S. 533.

69 Bd. 7, S. 753, Albert Einstein, 1979.

70 Bd. 7, S. 757/758.

71 Bd. 7, S. 917-919, 1990. 
«Seit Otto Hahn in einem Paraffinring das erste Atom spaltete und damit eine Energie freisetzte, deren Kraft er unterschätzte, hat sich das Weltbild verändert. Es gleicht verblüffend der politischen Lage, in der sich die Menschheit befindet. Wir leben in einem expandierenden Weltall, von dem wir annehmen, dass es durch eine gewaltige Explosion entstand, leer und doch angefüllt mit hundert Milliarden Galaxien, die sich manchmal durchdringen mit explodierenden und in sich zusammenfallenden und von Schwarzen Löchern aufgesaugten Sonnen, während immer neue in Gasnebeln entstehen, in einem Universum der Katastrophen, von dessen Zukunft wir nichts wissen, es kann sich endlos ausdehnen oder wieder zusammenstürzen, und auch die stabilen Systeme wie unser Sonnensystem können chaotisch werden, wie die Computer berechnet haben. Aber auch die Menschheit zeigt sich in einem ähnlichen Bilde. Wie das Weltall begann ihre neueste Geschichte mit der Explosion einer Atombombe und führte zum Wettrüsten und verbreitete die grosse Furcht; wie das Weltall expandiert auch die Menschheit, in absehbarer Zeit wird sie zehn Milliarden Menschen zählen. Wir bauen uns eine technische und ökologische Katastrophenwelt auf. Die Galaxis der Armut droht die unseres Wohlstandes zu durchdringen, die freie Marktwirtschaft beschwört Krisen herauf, Hochkonjunkturen dauern nicht ewig, sie saugt wie ein Schwarzes Loch die Ressourcen der dritten Welt auf. - Wir wissen ebenso wenig, wie unsere Zukunft sein wird, wie wir über die Zukunft des Weltalls wissen, in welchem wir leben. Wir können uns im Chaos verlieren oder in eine höllische Ideologie zusammenstürzen, und die atomaren Waffen sind erfunden, sie können nicht rückgängig gemacht werden.»

Könnte man die Kosmogonie und Menschheitsentwicklung in ihrer Zukunftsperspektive und Verknüpfung eindringlicher, bildhafter und eindrucksvoller gestalten als in diesen wenigen Sätzen?! Selbst ein so komplexes Geschehen wie die gegenseitige Durchdringung zweier Galaxien wird zu einer grossartigen Metapher genutzt, ebenso das Schwarze Loch, dem keine Materie und Strahlung mehr entrinnen kann.

Und eine andere Zukunftsperspektive an anderer Stelle ${ }^{72}$

«Die Sonne hat das Leben möglich gemacht und wird das Leben wieder verschlingen. Sie wird sich einmal aufblähen und die Erde zusammenschmelzen. Wie zu einem Klumpen Gold.»

Überall in seinen Werken bemüht F. D. kosmische und physikalische Bilder wie z.B.im Roman «Justiz», im Hörspiel «Das Unternehmen Wega», im «Verdacht», oder, grotesk-ironisch, im «Durcheinandertal». Gerade in letzterem Roman werden realistische Bilder mit Irrealem verwoben: So ist die Rede von Geschwindigkeiten, unermesslich grösser als die Lichtgeschwindigkeit, von Universum und Antiuniversum, die sich gegenseitig durchdringen,

«ohne sich berühren zu können, als lägen sie auf je einer der verschiedenen Seiten einer Möbius-Schlinge, auf der und in der die sich rasend drehenden Kugeln von Galaxien und Antigalaxien sich nie berührend bald auf der Schlinge, bald in der Schlinge schneller als Licht herumtollten .... ${ }^{73}$

Hier und im folgenden wird tatsächlich das physikalische, biologische und kosmologische Vokabular mit überbordender Phantasie und lustvoller Ironie durcheinandergebracht.

72 Bd. 5, S. 609, Midas, 1990.

73 Bd. 4, S. 903, Durcheinandertal. 
In dem Essay «Kunst und Wissenschaft ${ }^{74}$ verbindet F. D. kosmologische Details unseres Sonnensystems metaphorisch mit der Bio- und Noosphäre des Menschen. Da wird detailliert die Zusammensetzung unseres Sonnensystems aus Planeten, Monden, Gas- und Staubwolken, Planetoiden und Kometen beschrieben. Der Einfall wird mit der Sternschnuppe verglichen, die Vision mit einer Sonneneruption und die Noosphäre mit dem Sonnensystem, das sich ja auch aus einem sichtbaren und einem unsichtbaren Teil (Transpluto und viele Kometen) zusammensetzt.

Schon 1976/77 hat F. D. ähnliche Gedanken in «Überlegungen zum Gesetz der grossen Zahl» ${ }^{75}$ geäussert:

«Das Licht, das uns wärmt, entsteht im Sonneninnern. Es dauert - berechneten Wissenschaftler - 25000 Jahre, bis es uns erreicht, sich mühsam durch die gewaltige Masse des monströsen Gasballs kämpfend, um welchen wir kreisen ${ }^{76}$. So wird sich vielleicht der menschliche Geist gleichsam ins Menscheninnere zurückziehen, um dort seine schöpferischen Kernprozesse zu vollziehen.»

Und nun folgt eine apokalyptische Vision der Menschheit, wie er sie auch in seinen Bildern immer wieder dargestellt hat.

«Zum Schluss droht immer noch der Untergang der Menschheit. Nicht mehr als eine blosse Hypothese, technisch ist er möglich geworden. Für uns die schlimmste Wendung, aber für das Leben und für diesen Planeten die vielleicht beste .... Die Saurier mussten nach 60 Millionen Jahren (nach neuesten Forschungen 120 Millionen) Herrschaft abtreten, die zwei Millionen Jahre, die nach dem ersten Auftreten unserer Gattung vergangen sind, reichen möglicherweise schon ... wir sprachen auf der Welt vor und fielen durch.»

In dem Essay «Abschied vom Theater» (1990) im Nachlassband «Gedankenfuge ${ }^{77}$ fasst Dürrenmatt sein kosmologisches Wissen noch einmal zusammen und bringt es gleichzeitig auf den neuesten Stand.

«Eines der schönsten kosmischen Objekte ist der Krebsnebel, ein zartes blaues nebelhaftes Gebilde, rot umrandet und von weiss-rötlichen Bändern umschlungen. Es sind die Überreste einer Supernova, die sich mit einer Geschwindigkeit von 1100 Kilometern in der Sekunde ausbreiten, die Sonne, die explodierte, ist zu einem Neutronenstern zusammengefallen mit einem Durchmesser von etwa 20 Kilometern, sie enthält immer noch mehr als eineinhalb Sonnenmassen und dreht sich dreissigmal in der Sekunde um sich selber. Wir sehen den Krebsnebel von aussen, 5000 Lichtjahre (nach Störig 1500 LJ) von uns entfernt, ein mär-

74 Bd. 7, S. 539-541.

75 Bd. 7, S. 706 und 707.

76 Anmerkung: Nach H. J. Störig in Knaurs moderner Astronomie (1992) vergehen $10^{7}$ Jahre und mehr, bis das Licht, aus dem Kern der Sonne kommend, die Strahlungszone durchquert hat. Hat es dann die Photosphäre erreicht, trifft es in 8 Minuten auf der Erde ein. Interessanterweise hat F. D. im «Winterkrieg in Tibet», der 1990 eine Neufassung erhielt, die richtige Zahl, nämlich $10^{7} \mathrm{Jahre}$, eingesetzt, was einmal mehr beweist, mit welcher Sorgfalt er sich stets auf dem neuesten Stand wissenschaftlicher Erkentnis gehalten hat.

77 Gedankenfuge, S. 184/185. 
chenhaftes Gespinst; wären wir in ihm, wären wir nicht mehr.» - Der Mensch ist «das Resultat nicht nur unzähliger toter Lebewesen vor uns, sondern auch explodierender Supernovae wie im Krebsnebel, welche die Ursonne, die Planeten und uns mit jenen schweren Elementen verschmutzte, ohne die kein Leben möglich ist. ${ }^{78}$

In der Tat können bei den Fusionsprozessen in normalen Sternen nur Elemente bis zum Atomgewicht des Eisens entstehen. Nur bei den ungeheuren Energiemengen, wie sie bei Supernova-Explosionen freigesetzt werden, bilden sich die schwereren Elemente, ohne die unsere Planeten und damit die Entstehung von Leben auf dem Planeten Erde nicht möglich wäre.

«Und es ist nicht abwegig zu denken, dass der Urknall, mit dem unser Weltall begann, vielleicht das Ende eines zusammenstürzenden Weltalls war, das vor unserem existierte. Vielleicht ist der Tod der Vater aller Dinge.»

Hier schimmern Vorstellungen von der Kosmogonie durch, wie sie erst in allerjüngster Zeit (1991) von den Bonner Astrophysikern um Wolfgang Priester entwickelt und als Urschwung-Theorie der Urknall-Theorie entgegengestellt wurden. Die Urknall-Hypothese setzt im Punkt Null eine Singularität voraus, d.h. eine unendliche Dichte der Materie - der Abstand der Elementarteilchen untereinander ist Null -, eine unendliche Krümmung des Raumes und eine unendlich hohe Temperatur. Alle Gesetze über Raum und Zeit verlieren im Augenblick des Urknalls ihre Bedeutung. Diese Schwierigkeit umgeht die in den letzten Jahren von Priester und Blome aufgestellte neue Hypothese. Das Universum ist danach nicht durch einen Urknall entstanden, sondern besteht seit ewigen Zeiten als materie-freies Quantenvacuum, erfüllt mit Feldern hoher Energiedichte. Auch hier schrumpft der Raum allmählich bis auf eine minimale Ausdehnung von nur $10^{-25} \mathrm{~cm}$ zusammen, doch kommt es nicht zur Singularität; denn in einer Art Rückprall, bei dem sich Energie in Materie umwandelt $\left(E=m c^{2}\right)$, explodiert das Ganze und entwickelt dabei ähnliche Verhältnisse wie unmittelbar nach dem Urknall: Temperatur $10^{28} \mathrm{Grad}$, Materiedichte $2 \times 10^{76} \mathrm{~g} / \mathrm{cm}^{3}$ innerhalb von $10^{-33}$ Sekunden. Auch diese Werte sind unvorstellbar, aber mit den Naturgesetzen vereinbar und nicht wie bei der Singularität unendlich.

Auch St. W. Hawking - von F. D. häufig zitiert - ist in letzter Zeit von der Urknall-Theorie abgerückt, die ja die Entstehung von Raum und Zeit postuliert. Er spricht von Quantenfluktuationen, die zu immer neuen TochterUniversen führen (Eine kurze Geschichte der Zeit). Ähnliche Gedanken finden wir auch bei Dürrenmatt.

Werden, Vergehen und Neuentstehung des Kosmos in endloser Folge, von Ewigkeit zu Ewigkeit, wie die Astrophysiker neuerdings vorschlagen und mit

78 Anmerkung: Gedanken, wie man sie auch bei Hoimar v. Ditfurth in 〈Kinder des Weltalls〉 findet (Hamburg: Hoffmann und Campe, 1970). 
experimentellen Daten und Berechnungen wenigstens z.T. belegen, erinnern sehr stark an die mythischen Vorstellungen z.B. des Brahmanismus, Hinduismus, Buddhismus und Taoismus. Gemeinsam ist diesen im ersten Jahrtausend v. Chr. erdachten Weltbildern ein ewiges kosmisches Grundprinzip; im chinesischen Universalismus wird es Tao genannt, im indischen Brahmanismus und Hinduismus als Brahma bezeichnet, eine ewige aus sich selbst seiende Urkraft, die allem zugrundeliegt (Glasenapp). Raum und Zeit sind ohne Anfang und Ende. Der Kosmos unterliegt einem ewigen Kreislauf von Entstehen, Bestehen und Vergehen.

Zwischen den rationalen Naturwissenschaften und dem transzendentalen Denken der Religionen steht F. D. und entwickelt aus beiden Wurzeln seine grandiosen kosmischen Visionen.

Dürrenmatts frühe Liebe zu den Sternen kommt in der Beschreibung seiner Beziehung zu Max Frisch metaphorisch zum Ausdruck: Er vergleicht sich und Max Frisch mit dem Doppelgestirn

«... Kastor und Pollux, wobei es unklar ist, wer von uns beiden Kastor und wer Pollux darstellt, sind doch die beiden nur scheinbar ein Doppelgestirn: Kastor ist 42 und Pollux 32 Lichtjahre von uns entfernt ...»

Auch räumt er mit der weit verbreiteten Vorstellung auf, dass Kastor ein einzelner Stern sei; er ist vielmehr ein System von 7 Sonnen $^{79}$.

In märchenhaftem Gewande tauchen die Sternbilder schon in einem seiner frühen Stücke, «Ein Engel kam nach Babylon», auf:Andromedanebel, Mond, der blaue Sirius, die weisse Wega, die tosenden Cepheiden, das dunkle Feuer des Antares ${ }^{80}$. Von der wenigen Lyrik des Autors sind drei Gedichte in Versform den Gestirnen «Mond», «Antares» (roter Überriese) und «Siriusbegleiter» (Weisser Zwerg) gewidmet ${ }^{81}$.

Besonders in dem zuletzt genannten Gedicht zeigt sich, wie sehr F. D. der Faszination des Kosmos verfallen ist. In einfachen Versen schildert er das dramatische Schicksal einer Sonne, die nach einer langen stabilen Phase sich zu einem Roten Riesen aufbläht und grosse Energiemengen abstrahlt. Was übrig bleibt, ist ein Weisser Zwerg - «Erdenklein» - von ungeheurer Dichte. Alle Planeten und evtl. Leben auf ihnen sind ausgelöscht, die «schlimmstmögliche Wendung» im Sonnenschicksal ist eingetreten, eine Wendung, die

79 Bd. 6, S. 382/383. Anmerkung: Nach neuesten Erkenntnissen (Störig) der Astronomie ist Kastor ein Sternhaufen aus 6 Sternen und 50 Lichtjahre, Pollux hingegen 35 Lichtjahre entfernt.

80 Bd. 1, S. $457 \mathrm{ff}$.

81 Bd. 7, S. 616-618. 
dem dramaturgisch denkenden Autor besonders naheliegt. Dieses Unausweichliche, am Sternenschicksal beobachtet, findet man in Dürrenmatts Tragikomödien immer wieder.

In einem Liebesgedicht für Charlotte Kerr lässt er ihr «zu Ehren Galaxien, Supernoven» verpuffen ${ }^{82}$. Einen Brief unterzeichnet er mit «Leb wohl, Algol.» ${ }^{83}$ Algol ist der Teufelsstern, ein Stern, dessen Helligkeitsschwankungen durch «Sternfinsternis` - ein Stern tritt vor den anderen und verdeckt ihn - bedingt sind. Doch sein eigentliches Sternbild, mit dem er sich identifizierte, ist der Orion, der grosse Jäger der griechischen Mythologie, von Eos als Geliebter entführt. Ihn hatte er zunächst auf den nachtblauen Himmel um die Frau im roten Mantel gemalt, später aber wieder gelöscht ${ }^{84}$.

\section{Mathematik}

Über die Mathematik finden sich in Dürrenmatts Oeuvre zahlreiche Aussagen, die häufig mit erkenntnistheoretischen, philosophischen Fragen verknüpft sind. Deshalb sollen beide Themen in diesem Abschnitt gemeinsam behandelt werden.

Im Nachlassband «Gedankenfuge» ${ }^{85}$ schreibt F. D., dass die Denkgesetze der Logik der Mathematik immanent sind.

«Die Mathematik ist die unendliche Exekution der Logik.»

Schon der nächste Satz wirft Zweifel auf:

«Die Mathematik sagt nichts anderes aus als die von ihr konstruierten Begriffe, sie sagt nichts anderes aus als sich selber.»

Das ist sicher zu eng gefasst, wäre l'art pour l'art. F. D. widerspricht sich damit selber; denn im gleichen Essay ${ }^{86}$ widerlegt er diese Aussage:

«In der Physik wird die Mathematik transzendent, sie sagt etwas über die Physik aus und insofern die Physik etwas über die Natur aussagt, sagt die Mathematik etwas über die Natur aus.»

Auch im Essay «Vom Sinn der Dichtung unserer Zeit» ${ }^{87}$ schreibt er:

«In der Physik gewinnt die Mathematik einen bestimmten Inhalt, der ausser ihr liegt (in der Physik eben) ...»

82 Kerr, Ch.: Die Frau im roten Mantel. München/Zürich: R. Piper, 1992.

83 Ebenda, S. 106.

84 Ebenda, S. 107.

85 Gedankenfuge, S. 46/47.

86 Ebenda, S. 48/49.

87 Siehe das Kapitel Physik. 
«Die Mathematik ... kann nicht lügen. Sie kennt nicht das Urteil wahr oder unwahr, sie stellt nur fest, ob ihre Aussagen logisch oder unlogisch sind, richtig oder falsch ...» «Je mehr sich jedoch die Mathematik von der Sprache löst, ... desto schärfer bilden sich allmählich zwei Kulturen. Das Denken wird vom Gefühl getrennt. Die Denkgesetze erweisen sich als fähig, die Naturgesetze auszudrücken. Die Mathematik legt ihre Gesetze in die Natur hinein, und die Natur zwingt ihre Gesetze der Mathematik auf.»

Besser wäre die Formulierung, die Mathematik hat ihre Gesetze «nach der Natur> entwickelt (A. Dress, persönliche Mitteilung). Das Auseinanderdriften von abstraktem Denken und Gefühl ist sicher mit ein Grund dafür, dass das Gros der Menschen der Mathematik reserviert gegenübersteht. Im Zusammenhang mit Einstein stehen die Äusserungen über die euklidische Geometrie:

«Die euklidische Geometrie wird durch die nicht-euklidische erweitert, aber nicht erneuert.» 88

Später ${ }^{89}$ fasst er diese Aussage genauer:

«Es scheint mir nur eine Geometrie zu geben, die nicht-euklidische, von der die euklidische ein Sonderfall ist.»

In der Tat hat die Erweiterung notgedrungen zu einer Erneuerung geführt. In den gleichen Anmerkungen bringt F. D. im Zusammenhang mit der nichteuklidischen Geometrie einen erkenntnistheoretischen Aspekt ins Spiel, wenn er schreibt:

«Kants Auffassung der Mathematik ... hätte eine nicht-euklidische Geometrie durchaus zugelassen. Hätte er sie gekannt, hätte er möglicherweise den Begriff der menschlichen Anschauung erweitert; zu dieser gehört nämlich durchaus auch die mathematische abstrakte Anschauung, die mathematische Phantasie, die der Mathematik erlaubt, die sinnliche Anschauung zu durchstossen $»^{90}$.

Die Auseinandersetzung mit der Einsteinschen Relativitätstheorie führt im gleichen Kapitel zu einer Stellungnahme zu den Begriffen der Kausalität und des Determinismus. In der Newtonschen Physik gilt danach die Kausalität, z.B. für die Gravitation, in der Relativitätstheorie der Determinismus, der aus der «Krümmung des Raum-Zeit-Kontinuums durch das Vorhandensein der Materie» gefolgert wird. Diesem Grundgedanken kann man nur schwer folgen, da sich beide Begriffe ja nicht wesentlich voneinander unterscheiden. Kausalität ist die unmittelbare Folge von Ursache und Wirkung in einer Reaktionskette; Determinismus ist globale Kausalität. Vielleicht hat Dürrenmatt an dieser Stelle auch seine Schwierigkeiten gehabt und deshalb den Unterschied zwischen Kausalität und Determinismus als «ein nur im Bereich der Logik stattfindendes Scheingefecht» bezeichnet.

88 Bd. 3, S. 435, Achterloo.

89 Bd. 7, S. 761, Anmerkungen zu Albert Einstein.

90 Bd. 7, S. 760. 
In einem Gespräch mit Gerhard Vollmer im Oktober $1990^{91}$ kennzeichnet F. D. die Mathematik als eine der grössten Leistungen des menschlichen Gehirns und den Computer als ein mathematisches Hilfswerk,

«eine Erweiterung des Hirns, ... ein Denkzeug»

und er stellt die Frage:

«Inwiefern nimmt heute in der Naturwissenschaft die Mathematik die Stellung ein, die früher in der Geisteswissenschaft die Meatphysik innehatte? - Aber was ist nun die Mathematik, ist das ein Denken a priori? Man wundert sich immer, warum die Naturgesetze mathematisch sind. Es ist aber gar nicht anders möglich, weil die einzige Sprache, in der man sie darstellen kann, die Mathematik ist. - Die Mathematik führt eben die Anschauung weiter. Es ist natürlich grotesk, wenn man das noch Anschauung nennt. Aber es liegt in ihrem Wesen, auch diese Schallmauer ... zu durchstossen ... und dort fängt die Mathematik an, die Rolle zu übernehmen wie früher die Metaphysik ... Die Metaphysik ist Phantasie; ... aber die Mathematik durchstösst etwas, und es liegt eben auch ... in der Fähigkeit des Hirns selbst. - In der heutigen Mathematik wird eben das Unanschauliche denkbar.»

Diese Gedanken kommen dem hypothetischen Realismus (Campbell), den Konrad Lorenz ${ }^{91 a}$ vertritt, recht nahe. Danach ist unsere Denkordnung eine Nachbildung der Naturordnung. Denken und Erkennen sind evolutiv entstanden. Die apriorischen Wahrnehmungs- und Denkkategorien sind Produkte einer biologischen Entwicklung.

Auch kausales Denken hat offensichtlich eine angeborene Grundlage, ist in unseren Denkstrukturen genetisch fixiert. Es geht der Erfahrung voraus und ist damit psychologisch a priori. Nach Kant ist unsere Erkenntnis über die Welt (Raum, Zeit, Kausalität) a priori; nach Konrad Lorenz ist sie nur in der Individual-Entwicklung a priori (von der Stammes-Entwicklung und damit genetisch vorgegeben), in der Stammes-Entwicklung hingegen a posteriori.

Die apriorischen Inhalte des Einzelmenschen sind die aposteriorischen Erfahrungen der Evolution. Im Gespräch mit Gerd Vollmer blitzt dieser erkenntnistheoretische Gedankengang noch einmal auf, wenn F. D. sagt:

«Dass die Natur zwei Gesichter hat, dass man einerseits das Licht als Welle und andererseits als Partikel feststellen kann - ist das nicht begründet im menschlichen Geist? In unserer Denkweise?»

Man kann diese Frage nur bejahen.

Die Beziehung der Mathematik zur Physik ist ein viel besprochenes Thema bei Dürrenmatt. In dem Essay «Vom Sinn der Dichtung in unserer Zeit» (1956) setzt er sich mit dieser Thematik ausführlich auseinander ${ }^{92}$. 
«Da aber die Mathematik das Bild nicht benötigt, an sich sein kann als ein Operieren mit reinen Gedankendingen, andererseits eine sichere immanente Logik besitzt, ist nun auch die Physik der Genauigkeit des Denkens zuliebe immer mehr ... dazu übergegangen, ebenfalls die Anschauung, das Bild, das Modell endlich, fallen zu lassen. Sie stellt die Verhaltensweisen der Natur nicht nur mathematisch dar, sie versteht sie auch mathematisch. - Nun tröstet man sich im allgemeinen damit, dass dieser Weg aus der Sprache heraus in die Begriffswelt der Mathematik nur im naturwissenschaftlichen Denken stattfinde, jedoch nicht in der Philosophie oder in den sogenannten Geisteswissenschaften, die sich immer noch in der Domäne des Wortes aufhalten. Doch möchte ich hier einmal den Verdacht anmelden, ob nicht die Form der heutigen Philosophie die Naturwissenschaft sei, ob wir uns nicht einer Täuschung hingeben, wenn wir glauben, immer noch die alte Philosophie des Wortes in irgendeiner Form aufrechterhalten zu können, ob es nicht einfach so sei, dass wir bei Einstein und Heisenberg die Ansätze einer neuen Philosophie finden und nicht bei Heidegger.»- «Die Physik, die Naturwissenschaft ist durch ihre notwendige Verbindung mit der Mathematik weitgehend dem Verständnis des Nichtphysikers entrückt, das heisst, dem Verständnis der überwiegenden Anzahl der Menschen.»

Hier werden für die Ohren der Geisteswissenschaftler geradezu ketzerische Gedanken geäussert, die nur vor dem Hintergrund zu verstehen sind, dass F. D. von der Philosophie und Naturwissenschaft her kommt. Diese Verbindung beider Disziplinen ist nicht nur notwendig, sondern auch für beide vorteilhaft, wenn sich auch die Philosophen auf keinen Geringeren als Kant berufen können, der die Naturwissenschaften von der Philosophie trennte. Doch tempora mutantur et nos mutamur in illis.

In «Kunst und Wissenschaft», $1984^{93}$, wird unter Bezugnahme auf Platon der Gedanke erneut aufgegriffen:

«Die Vermutung, die Ideen seien Zahlen, ist eine Vorahnung der heutigen Physik ... die Physik ist ohne Mathematik nicht denkbar. - Vielleicht sind nur mathematische Ideen Ideen, darum hat denn auch die Mathematik etwas Expandierendes. Ihren Ideen sind keine Grenzen gesetzt, eine Vollendung der Mathematik ist undenkbar.»- «Nur das ist physikalisch, was sich mathematisch ausdrücken lässt, die formale Seite der Natur, die Natur als Idee.»

Hier schimmern Gedanken aus Platons Ideenlehre durch, hier entfernt sich F. D. vom oben beschriebenen hypothetischen Realismus, hier läuft er Gefahr, dem folgenschwersten Irrtum des Idealismus zu erliegen, an der Realität der Aussenwelt, welche die Physik erforscht, zu zweifeln. Auch der Vorstellung, die Physik gehe das Wagnis ein, «die Wirklichkeit den mathematischen Ideen unterzuordnen», muss widersprochen werden: Die Physik nutzt die mathematischen Ideen als Erklärungsmöglichkeit der beobachteten und erforschten Phänomene. Sehr richtig beurteilt F. D. die Rolle Kants in diesem erkenntnistheoretischen Prozess. Kant hat die Metaphysik abgeschafft, «zertrümmert».

93 Bd. 7, S. 548/549. 
«Er erklärte die Mathematik ausschliesslich für die Erfahrung brauchbar und für diese die Metaphysik unbrauchbar. Er teilte die Welt in einen durch die Form unserer Vorstellung und die Kategorien unseres Denkens erfahrbaren physischen und in einen grundsätzlich jenseits jeder möglichen Erfahrung liegenden Bezirk ein, jenen des Dings an sich .... ${ }^{94}$

Kant erkannte hier, weit vorausblickend, dass unsere Erkenntnismöglichkeiten entscheidend geprägt sind durch die Strukturen und Denkkategorien unseres Gehirns. Und in dem Vortrag Albert Einstein ${ }^{95}$ :

«Kant ersetzt die Metaphysik durch die Mathematik und die Physik. Für Kant ist der Schluss der Metaphysiker vom Denken auf das Sein, das hinter der Erfahrung liegt, unmöglich ... Demgegenüber ist die Mathematik möglich, indem sie nur sich ausdrückt, sie ist eine apriorische Begriffskonstruktion, die nur eine innere Widerspruchsfreiheit erfordert.»

In dem gleichen Vortrag setzt F. D. sich ebenfalls vehement für die Verbindung vom Mathematik, Naturwissenschaften und Philosophie ein:

«Mathematik, Naturwissenschaften und Philosophie sind heute derart ineinander verflochten, dass sich auch Laien mit diesem gordischen Knoten befassen müssen. Denn überlassen wir die Physiker, die Mathematiker, die Philosophen sich selber, treiben wir sie endgültig in die Ghettos ihrer Fachgebiete zurück, wo sie hilflos und unbemerkt den Raubzügen der Techniker und Ideologen ausgeliefert sind ... ${ }^{96}$

Das Kapitel kann nicht abgeschlossen werden, ohne die erkenntnistheoretisch entscheidende Frage zu erwähnen, die hinter vielen Äusserungen Dürrenmatts steckt: Ist das, was ich sehe, die Wirklichkeit, oder setzt sich die Wirklichkeit erst in meinem Kopf als Wirklichkeit zusammen? ${ }^{97}$ Auch in dem Essay «Das Hirn» taucht dieser für F. D. so typische Grundzweifel auf:

«Ist das Hirn meine Fiktion, die ich schreibe, oder bin ich die Fiktion des Hirns, die das 〈Hirn〉 schreibt?» - «Doch auch wenn vom Hirn ... diese Welt zusammengeträumt wäre, so ist sie doch unsere Welt, sei sie nun wirklich oder nur möglich ...»

Das alte erkenntnistheoretische Problem: Idee oder Realität, das schon bei Platon im Höhlengleichnis angesprochen wird - von F. D. in seinem Essay «Das Haus» zitiert $^{98}$ - ist unentscheidbar. Ist die Idee, dass die alltäglichen Gegenstände unserer Umwelt erst durch unser Erleben Realität erhalten, wie der Idealismus lehrt, für F. D. das Entscheidende, oder die Vorstellung des hypothetischen Realismus, für den alle Erkenntnis auf Wechselwirkungen zwischen Subjekt und Objekt beruht, die beide gleichermassen wirklich sind? Dürrenmatt spielt mit beiden Auffassungen, ohne sich für eine zu entscheiden. Wenn er in dem angeführten Essay weiter schreibt:

94 Bd. 7, S. 583/584.

95 Bd. 7, S. 742.

96 Bd. 7, S. 723.

97 Gedankenfugen, S. 172, Abschied vom Theater.

98 Bd. 6, S. 437/438. 
«... die vollständige mathematische Durchdringung der Wirklichkeit ... wird nicht die Wirklichkeit sein, sondern die mathematische Interpretation der Wirklichkeit»

so kann man ihm nur zustimmen. Aufgrund der «Beschränktheit unseres Wahrnehmungsapparates können wir immer nur Teilaspekte der Wirklichkeit erfassen, nie die ganze Wirklichkeit, wenn auch mit Hilfe der Prothesen, die das menschliche Gehirn geschaffen hat, der Ausschnitt ständig vergrössert wird. Zum Abschluss dieser Überlegungen ein Satz Alexander I. Wittenbergs:

«Letzte und absolute Erkenntnis ist uns nicht zugänglich - der tiefste Grund hierfür liegt nicht in einer Schwäche unseres Erkenntnisvermögens, sondern bereits in der Tatsache, dass unsere begrifflichen Mittel sich jeder absoluten Sicherung entziehen.» ${ }^{99}$

Das gilt auch für die Mathematik.

\section{Biologie}

Die biologischen Themen treten in Dürrenmatts Oeuvre erst in den 80er Jahren auf, wenn man einmal von einer kurzen metaphorischen Notiz im Mitmacher 1972/73 absieht, besonders aber in seinem letzten Lebensjahr 1990.

Vor allem die Evolution und der komplexe Aufbau des menschlichen Gehirns mit seinen grandiosen Leistungen faszinierten ihn. So ist es kein Zufall, dass er dem Gehirn eigens einen Essay ${ }^{100}$ gewidmet hat.

In diesem Essay wird die Schöpfung und ihre Evolution in einer absurden, irrwitzigen Vision beschrieben. Am Anfang war nicht das Wort oder die Tat, auch nicht Gott oder Urknall, sondern das Gehirn als dimensionsloser Punkt, als Singularität, das, zunächst leer, Schritt für Schritt das Sein erdenkt und damit erschafft. Das Absurde an dieser Vision ist, dass ein menschliches Hirn, das Endprodukt einer 31/2 Milliarden Jahre währenden biologischen Evolution in einem 15 Milliarden Jahre alten Kosmos, am Anfang steht. Indem es die Entwicklung des Kosmos, des Lebens und seine eigene Evolution erdenkt - das ist subtil und kenntnisreich von F. D. beschrieben -, lässt der Autor zwei Entwicklungsstränge parallel nebeneinander ablaufen: den des Hirns und damit der Idee, und den der erdachten Aussenwelt, der Realität. Beide sind umeinander geschlungen wie eine Doppelhelix und miteinander verbunden wie die beiden DNA-Stränge durch Wasserstoffbrückenbildung. Es ist die Faszination über die Möglichkeiten und vollbrachten Leistungen des mensch-

99 Wittenberg, A. I.: Vom Denken in Begriffen, S. 359. Basel/Stuttgart: Birkhäuser, 1968.

100 Bd. 6, S. 539ff. Das Hirn, 1990. 
lichen Gehirns und seiner Geistes- und Kulturgeschichte, der Welt III nach Popper, die F. D. zu diesem komplizierten Gedankenspiel veranlasst haben könnte. Es ist aber auch unübersehbar die platonische Ideenlehre - denn die Idee hat in diesem Essay die absolute Priorität-, die dem Gedankenspiel zugrundeliegt, auch wenn der ständig nagende erkenntnistheoretische Zweifel an der Richtigkeit dieser Lehre nicht zu übersehen ist.

Dieses Hirn, das F. D. fingiert, ist, wie er ausdrücklich betont, ein menschliches Gehirn,

«das rätselhafteste und komplizierteste Gebilde, das wir kennen, eine Galaxis von Schaltelementen, von Neuronen, untereinander vernetzt, eingeteilt in Kleinhirn, Stammhirn, Zwischenhirn, Grosshirn ...»

An anderer Stelle ${ }^{101}$ beschreibt er das menschliche Hirn

«... wunderbarer noch als Gott, den es sich auszudenken vermag, und der Weltraum, den es sich vorzustellen versucht.»

Oder in Abendstunde im Spätherbst ${ }^{102}$

«... das Hirn ist für mich das Komplizierteste, was wir von der Welt kennen. Eine Sonne ist gegenüber dem Hirn etwas ungeheuer Primitives. - Für mich ist das Hirn etwas viel Grandioseres als z.B. ein Stern oder ein Quasar.»

Da es im Punkt Null keine Aussenwelt gibt, wird das Hirn sich zunächst nur selbst fühlen und voller Angst und Entsetzen sein über die Leere; denn es ist nichts in ihm gespeichert. Doch das Werkzeug, das «Denkzeug», zum Denken und Speichern ist komplett vorhanden. Dabei kommt es zum Ionenfluss und zu elektrischen Strömen, die das Hirn fühlt. Doch all das enthält noch keine Information.

«Es beginnt zu erfühlen, es erfühlt die Zeit ... Es erfühlt die Gegenwart, durch die ein Impuls fliesst und dann der nächste ... dann die Vergangenheit ... das Gedächtnis bildet sich ...»

Es folgt das Erfassen einer binären Zahlenreihe, das Erfassen von Rhythmen und Erfühlen von Tönen, die sich zu Musik verdichten. Die anfänglich beherrschende Angst weicht der Freude, eine Ordnung gefunden zu haben. $\mathrm{Zu}$ Angst und Freude gesellen sich im Laufe der Evolution die anderen Gefühlsqualitäten, Trauer, Neugier, Zorn, Ohnmacht, Langeweile, Wut. Nach langer Zeit endlich entdeckt das Hirn sich selbst, das Ich; das Bewusstsein seiner selbst ist erwacht. Vor dem Ansturm der Gefühle klammert das Gehirn sich wieder an die Zahlen, die ihm als erste Schutz vor der Angst geboten haben. Das Hirn erfindet die Mathematik, findet in ihr aber «kein echtes Gegenüber». Denn die Mathematik ist seinem Denken immanent.

101 Bd. 6, S. 536, Vinter, 1990.

102 du, Heft Nr. 1, Januar 1991. Abendstunde im Spätherbst. 
An diesem Punkt nähert sich F. D. den Vorstellungen des hypothetischen Realismus. Der nächste entscheidende Schritt, den das Hirn vollzieht, ist, aus sich selbst herauszutreten, das Aussen, das Andere zu entdecken. Und nun beginnt der eigentliche Schöpfungsakt. Das Hirn erschafft, denkend, Materie, Raum, ganze Welten und schliesslich das Leben.

«es überdenkt die 30 Aminosäuren, durchaus nicht nur mathematisch, das wäre bei der ungeheuren Anzahl von möglichen Variationen (einer 1 mit 190 Nullen) unmöglich, hat es doch nur 1 Billion Jahre Zeit, $10^{17}$ Sekunden, ... sondern selektierend, spielerisch ...» ${ }^{103}$

Mit den Begriffen «selektierend, spielerisch» ist der Vorgang der Selbstorganisation der Materie nicht hinreichend beschrieben. Erst das zyklische Zusammenwirken von autokatalytischen und selbstinstruktiven Verbindungen mit einem Kopplungsprotein führte zum Hyperzyklus und von dort durch scharfe Selektion zum genetischen Code, Voraussetzungen für die Entstehung der Urzelle, wie M. Eigen ${ }^{104}$ zeigen konnte. Durch die «Ungenauigkeit bei der Reproduktion» kommt es zur Evolution von der Urzelle zu den verschiedenen Arten. Der Tod wird ins Leben integriert. Auch im Abschied vom Theater, $1990^{105}$, schreibt F. D.:

«... ohne den Tod keine Evolution, ohne den Tod wären wir nichts als ein Schorf sich ständig teilender Einzeller, die Erde bedeckend ...»

Gleiche Gedanken äussert er in «Abendstunde im Spätherbst» ${ }^{106}$, in den 1990 geführten 5 Gesprächen «Über die Grenzen» ${ }^{107}$ und im «Prometheus»108,109.

Doch zurück zum Essay «Das Hirn». Es folgen die Beschreibung der Evolution der Säugetiere und die Entwicklung des Menschen, seine Sozialisation, die Entstehung der Sprache. Das Bewusstsein des eigenen Todes wird zum Ursprung der Kultur.

103 Anmerkung: Der Zeitraum der Evolution des Lebens ist mit $10^{12}$ Jahren zu hoch gegriffen. Er liegt bei 3,8 $\times 10^{9}$ Jahren. Das Leben arbeitet nicht mit 30, sondern mit 20 Aminosäuren, die in Nukleinsäure-Tripletts kodiert sind. Wenn man die möglichen Variationen bei der Bildung eines Protein-Moleküles berechnen will, muss man sich auf eine bestimmte Molekülgrösse einigen: z.B. ein Protein mit 100 Aminosäuren - das entspricht einem kleinen Protein, dem Cytochrom-Molekül. Von ihm können $10^{130}$ verschiedene Sequenzen gebildet werden. Dazu würde das Alter des Kosmos, das z.Z. auf 15 Milliarden Jahre $=5 \times$ $10^{17}$ Sekunden veranschlagt wird, nicht ausreichen. Die Konsequenz: Durch Zufall allein könnte ein so komplexes Funktionssystem, wie es die Proteine darstellen, nicht entstehen.

104 Eigen, M.: Stufen zum Leben. München/Zürich: R. Piper, 1987.

105 Gedankenfuge, S. 185.

106 du, Heft 1, Januar 1991.

107 Über die Grenzen, S. 13.

108 Gedankenfuge, S. 32.

109 Anmerkung: Der Zusammenhang zwischen Tod und Evolution kann so nicht gesehen werden. Evolution hat es auch bis zum Einzeller schon gegeben. Das Darwinsche Prinzip bestimmt die Evolution von der unbelebten zur belebten Materie, von der Urzelle bis hin zur 
Wenn Dürrenmatt über die Herkunft und Entstehung im allgemeinen und des Menschen im besonderen nachdenkt, weist er darauf hin, dass wir

«das Resultat nicht nur unzähliger Lebewesen vor uns, sondern auch explodierender Supernovä wie die im Krebsnebel ...»

$\operatorname{sind}^{110}$.

«Ich werde mir bewusst, dass ich aus Materie komponiert bin, deren innerste Strukturen, die wir etwa Protonen, Neutronen, Elektronen und Quarks neuerdings nennen, seit dem Urknall bestehen, sodass eigentlich die Materie unvergänglich und das Leben, der Geist, das Bewusstsein, der Intellekt, der diesen Gedanken denkt, das Ich das Einmalige und darum Vergängliche ist.»

Wir sind «Kinder des Weltalls`, wie Hoimar von Ditfurth es nennt, und vereinigen Mikro- und Makrokosmos in uns.

Schon im «Mitmacher», 1972/73 ${ }^{111}$, weist F. D. auf die Ursynthese von Aminosäuren in der Ursuppe unter dem Einfluss der Uratmosphäre hin, wie sie Stanley Miller experimentell nachvollzogen hat:

"Wer ein Gemisch aus Methan, Wasserdampf, Ammoniak und Wasserstoff einer elektrischen Ladung aussetzt, erhält Aminosäure, den Grundstoff des Lebens.»

In den Gedanken über seine Komödie «Der Mitmacher», $1976^{112}$, widmet er einen ganzen Abschnitt dem Thema Zufall und Evolution. Die Entstehung des Lebens, von der Uratmosphäre, der Ursuppe, der Bildung der ersten grösseren Molekülverbände bis zur Urzelle in ihrer jetzigen chemischen Zusammensetzung, ist nach F. D.'s Vorstellung nichts als eine Folge von

«ganz und gar phantastischen Zufällen.»

Dabei übersieht er die Gesetzmässigkeiten, die Notwendigkeiten der Biochemie, wie z.B. die Komplementarität der Nukleinsäuren als eine diesen Verbindungen inhärente Eigenschaft. Oder die Selbstorganisationsmechanismen der Materie, wie M. Eigen im Hypercyclus nachgewiesen hat ${ }^{113}$. Vor allem aber bleibt bei seinem Eilmarsch durch die Evolution der entscheidende Begriff der Information unberücksichtigt. Mit anderen Worten: Naturgesetze steuern den Zufall (M. Eigen).

Differenzierung der verschiedenen Einzeller. Die Einführung der geschlechtlichen Vermehrung zu einem Zeitpunkt, da die Evolution infolge zu gut funktionierender Repair-Mechanismen ins Stocken geraten war, ist der entscheidende Schritt zur Weiterentwicklung der Arten. Um eine hinreichend schnelle Durchmischung des Genpools zu erreichen, musste die Lebensdauer der bis dahin unsterblichen Lebewesen begrenzt werden: Der Tod des Individuums wurde erfunden > und genetisch fixiert. Auf diese Weise kam wieder hinreichend Variation in das System, die Evolution zu den höheren Arten konnte beginnen. Es ist also primär nicht der Tod, sondern die geschlechtliche Vermehrung und in ihrem Gefolge der programmierte individuelle Tod, welche die Evolution weiterbrachten.

110 Gedankenfuge, S. 185, Abschied vom Theater, 1990.

111 Bd. 3, S. 58.

112 Bd. 7, S. 206-212.

113 Eigen, M.: Stufen zum Leben. 
Im weiteren Verlauf des Essays betrachtet F. D. die Entwicklung des Menschen vom Proconsul über die Praehominiden zum homo sapiens ironisch und die heutige Situation kritisch:

«... sie grenzt ans Kriminelle, die Schuldfrage wird noch untersucht, der Verkehrsunfall ist zu gigantisch»

(exponentielle Bevölkerungsexplosion trotz Pille, Umweltverschmutzung, Aufbrauchen der Ressourcen, Hungersnöte in nie gekannten Dimensionen usw.).

«Sicher kommt der Evolution eine gewisse Schuld zu ...»

- diesen Satz kann man nicht unterschreiben. Der Mensch hat mit Erreichen der Stufe eines sich seiner selbst bewussten Lebewesens die Gesetze der natürlichen Selektion für sich und einen Teil der mit ihm eng verknüpften Natur weitestgehend ausser Kraft gesetzt. Eine geistige Evolution mit analogen Methoden - Mutation, Selektion, Optimierung - müsste die natürliche Evolution fortsetzen. Sie müsste sehr viel schneller ablaufen als die natürliche Auslese. Die Alternative wäre der Untergang der Menschheit in einer (selbstverschuldeten?) globalen Katastrophe.

Mehrfach kommt die Fragwürdigkeit des Menschen zur Sprache.

«Als Tier ist der Mensch das Resultat einer etwa eine Milliarde Jahre langen Evolution.» ${ }^{114}$

«Erst in den letzten zwei, vielleicht drei Millionen Jahren haben wir uns langsam zum Menschen entwickelt.» ${ }^{115}$

«... wie Schatten aus der Vergangenheit bedrängen uns Triebe, die unser Intellekt nur unvollkommen zu steuern weiss. Aus evolutionärer Pfuscherei entstanden, stürzen wir, in den Entropie-Strom getaucht, dem wahrscheinlichsten Zustand entgegen, in ein totes Universum hinein ...»"116

Ganz ähnliche Gedanken werden im Gespräch mit Gerhard Vollmer 1990 geäussert ${ }^{117}$ :

«Nun hat sich die menschliche Evolution so entwickelt: Das Hirn ist dem Menschen davongaloppiert.»

Oder:

«Der Mensch hält eigentlich sein eigenes Hirn gar nicht mehr aus. Das ist für mich ein Zeichen einer biologischen Krise».

114 Anmerkung: muss heissen, 3,8 Milliarden Jahre.

115 Bd. 7, S. 904.

116 Gedankenfuge, S. 33, Prometheus, 1992.

117 du, Heft 1, Januar 1991. 
Im weiteren Verlauf des Gespräches kommt Vollmer zu der wichtigen Unterscheidung von biologischer Evolution des Gehirns und geistiger Evolution. F. D. spürt sehr deutlich das Auseinanderdriften von genetisch verankerten, fast statischen Instinkten einerseits und dem Intellekt andererseits, der mit, fast könnte man sagen, Lichtgeschwindigkeit in immer neue Räume vorstösst und sich weiterentwickelt, auch wenn das im Gespräch nicht ganz so prägnant zum Ausdruck kommt, wie das in einem wieder und wieder überarbeiteten Essay der Fall wäre.

«Ich glaube eben, dass das Gehirn (biologisch) gleich geblieben ist: es schleppt Urängste, es schleppt Gefühle, es schleppt Aggressionen mit sich. Was ich behaupte, ist, dass der Mensch langsam im Gegensatz steht zu seinen Urinstinkten ... in einem Gegensatz zu sich selbst.»

In der Tat trifft das den Kern unserer heutigen Situation: die Schere zwischen der genetisch fixierten Welt des Emotionalen, das den über sehr lange Zeiträume wirkenden Gesetzen der biologischen Evolution unterliegt, und der Welt unseres Intellektes, die diesen Gesetzen nicht gehorcht, sondern ihre eigenen, rasant wirkenden und sich ändernden Gesetze entwirft, öffnet sich immer mehr. Das ist das Dilemma unserer Zeit. Ein Produkt dieses Intellekts ist die Technik, die mehr und mehr Teil der menschlichen Evolution wird.

«Was die Dinosaurier, die bis jetzt erfolgreichsten Lebewesen, während der vielen Millionen Jahre ihrer Herrschaft aus ihrem Skelett schufen, indem sie ihre Knochen umfunktionierten, so dass die Bestien die Luft, das Land und das Meer beherrschten, schafft der Mensch durch die Prothesenwelt seiner Maschinen.»

(Vielleicht sollte man noch ergänzen: in wenigen Jahrhunderten.)

«Auch sie gehören zur menschlichen Biologie, die Technik durchwächst den Menschen, wird ein Teil von ihm, - von seinem Leib und von seinem Geist.» ${ }^{118}$

Im Essay «Varlin ${ }^{119}$ heisst es:

«... das Leben ... eine Rebellion gegen die Entropie, gegen den natürlichen Hang der Materie, in ihre natürliche Ordnung einzuspuren, in die Unordnung.»

Diese Beschreibung der Beziehung des Lebens zum 2. Hauptsatz der Thermodynamik findet sich in ähnlicher Weise in dem Essay «Der Winterkrieg in Tibet» ${ }^{120}$ :

«... ein denkendes Wesen ist das unwahrscheinlichste, weil komplizierteste Wesen des Weltalls. Dieses Wesen scheint dem Weltgesetz, der Entropie zu widerstehen ...»- «Der Tod und die Entropie sind das gleiche Weltgesetz, sie sind identisch.»

118 Bd. 7, S. 552/553, Kunst und Wissenschaft, 1984.

119 Bd. 7, S. 482.

120 Bd. 6, S. 113. 
Dieser Gedanke weist auf E. Schrödinger hin. Korrekterweise müsste es heissen, die Entropievermehrung in einem geschlossenen System ist das Weltgesetz. Im offenen biologischen System wird die Entropie-Zunahme, gleichbedeutend mit der Tendenz des Systems in einen Gleichgewichtszustand überzugehen, kompensiert durch Energiezufuhr von aussen, die das lebende System fernab vom Gleichgewichtszustand hält. Denn Gleichgewicht bedeutet Tod.

Im Gespräch mit Michael Haller im Dezember 1990, kurz vor seinem Tode, vertritt F. D. noch einmal seine pessimistische Auffassung von der Rolle des Menschen in der Evolution:

«Heute wissen wir, dass der Mensch biologisch sehr viel mehr bestimmt ist, als man früher angenommen hat. Die wissenschaftliche Erkenntnis über die Genetik der Lebewesen hat uns alle Illusionen genommen. Und hat etwas Lähmendes.» - «Der Mensch als Gattungswesen ist in eine tiefe Krise geraten, ist krank.» ${ }^{121}$

Im gleichen Band lesen wir im Gespräch mit Sven Michaelsen ${ }^{122}$ :

«Der Mensch ist nicht imstande, gemäss seinem Wissen zu handeln, der Intellekt greift nicht. Das nenne ich das Apokalyptische.» - «Deshalb muss die Literatur fragen, ob die Menschheit nicht in einer evolutionären Krise steckt und auf ihr Ende zugeht.»

Diese apokalyptische Vision findet sich auch in Dürrenmatts Bildern in vielen Variationen. Sie ist die Konsequenz seines Denkens, immer von der schlimmst-möglichen Wendung des Geschehens auszugehen. Leider deckt sie sich weitgehend mit der Realität.

In diesem Zusammenhang ist auch die Frage zu sehen, welches das Ziel und der Zweck der Evolution ist, eine teleologische Frage, die für die Geisteswissenschaft charakteristisch ist. Der Naturwissenschaftler kann sich allenfalls dahingehend äussern, dass ein Optimierungsprinzip vorhanden ist, das für die Anpassung des Lebens an die jeweils neuen Umweltbedingungen sorgt. F. D. greift bei der Beantwortung dieser Frage auf Teilhard de Chardin zurück:

«Ich stimme Teilhard zu: Das Ziel der Evolution ist nicht das Himmelreich, sondern die Entfaltung alles Möglichen, praktisch: das Ausdifferenzieren alles Lebenden. Evolution bedeutet immer mehr Komplexität.» ${ }^{123}$

Das steht in einem gewissen Widerspruch zu seiner früheren Äusserung, dass die Natur nicht nach Zwecken handelt; sie ist sich selbst genug. Einen ganz ähnlichen Gedanken findet man bei Thomas Mann im Felix Krull:

121 Über die Grenzen, S. 114 und 111.

122 Ebenda, S. 19.

123 Ebenda, S. 69. 
«Sie (die Natur) denkt sich gar nichts, und auch der Mensch kann sich nichts bei ihr denken, sondern sich nur verwundern über ihren tätigen Gleichmut...» ${ }^{124}$

Während F. D. in dem Essay «Das Hirn» von einer grandiosen Fiktion ausgeht, beschreibt er an anderer Stelle sehr konkret die Leistung des Gehirns beim Sehvorgang:

«Sehen ist mit den Augen fühlen. Mein Auge sieht nicht, es leitet durch fotochemische Prozesse einen Frequenzbereich der elektromagnetischen Wellen in jene Teile des Grosshirns, wo sie in die Empfindung von Farben umgewandelt werden: eine sehr oberflächliche Darstellung eines komplizierten Vorgangs. Die Farbe ist damit etwas Objektives und Subjektives zugleich, eine Frequenz, die sich genau angeben lässt, und eine Empfindung, die subjektiv bleibt ...» «Das Bild der Aussenwelt, das in der sog. Sehrinde entsteht, wird auch erkannt: wir sehen nicht nur Farben, wir sehen auch Formen und Gegenstände.»125

Im Nachlassband «Gedankenfuge» ${ }^{126}$ wiederholt F. D. den Gedanken mit fast den gleichen Worten.

\section{Schlussfolgerungen}

Wenn man selbst mit Leidenschaft die Entwicklung der Naturwissenschaften erlebt und verfolgt, dann spürt man sehr bald bei der Lektüre des Dürrenmattschen Oeuvres die geistige Verwandtschaft. Man taucht tief ein in das Werk dieses aussergewöhnlichen Schriftstellers, um die naturwissenschaftlichen Schätze vom Meeresgrund an die Oberfläche des allgemeinen Bewusstseins zu holen. Dabei wird man gezwungen, auch sein eigenes Wissen zu vertiefen und neue Bezüge herzustellen; denn das Wissen F. D.'s ist umfassend, seine Verknüpfungen naturwissenschaftlichen Denkens mit Philosophie, Theologie und Geschichte sind originell und aufregend.

F. D. ist ein assoziativer Querdenker. Naturwissenschaft wird nicht um ihrer selbst willen beschrieben, sondern kritisch beobachtet und in den grossen Zusammengang gestellt, in sein Weltbild eingebaut. Andererseits prägt sie sein Weltbild entscheidend und regt ihn zu immer neuen Assoziationen an.

Gerade weil er sich des Stilmittels der Fiktion und der Vision bedient, weil er das Apokalyptische in Wort und Bild herausarbeitet, ja, weil sein Denken bei aller Schärfe des Verstandes bildhaft ist, gelingt es ihm, auch hochkomplexe Zusammenhänge verständlich darzustellen - erinnert sei nur an die

124 Mann, Th.: Bd. VIII, 7. Kapitel, S. 576f. Frankfurt/M.: S. Fischer, 1960.

125 Bd. 7, S. 530ff.

126 Gedankenfuge, S. 51. 
oben zitierte gegenseitige Durchdringung von zwei Galaxien, die sich in der Durchdringung von Armut und Wohlstand metaphorisch widerspiegelt ${ }^{127}$.

Ich hoffe, anhand vieler Beispiele belegt zu haben, dass meine eingangs aufgestellte These richtig ist: Das naturwissenschaftliche Denken in Dürrenmatts Werk ist nicht marginal, sondern unlösbar verschränkt mit Philosophie, Politik und Gesellschaftskritik.

Naturwissenschaftliches Denken ist eine für F. D. spezifische Denkweise. Sein Wissen ist universal, seine Dimension das Universum und die Menschen ihn ihm, Produkt einer kontinuierlichen Evolution. Daraus leitet sich sein kopernikanisches Weltbild ab, seine Absage an Gott, der in diesem naturwissenschaftlich geprägten Weltbild keine Notwendigkeit darstellt.

Spätestens hier stellt sich die Frage, welche Funktion, welche Bedeutung haben die naturwissenschaftlichen Themen in seinen Texten und Bildern? Er selbst gibt uns die Antwort, und damit schliesst sich, an die Einleitung anknüpfend, der Kreis:

«Die Welt dramaturgisch in den Griff zu bekommen, das geht heute ohne die Beschäftigung mit der (Natur-)Wissenschaft überhaupt nicht. - Die zweite, die naturwissenschaftliche Kultur, ist heute das Entscheidende.» (Interview mit Sven Michaelsen am 7.12.1990).

An anderer Stelle weist er auf den gordischen Knoten hin, zu dem Mathematik, Naturwissenschaften und Philosophie verflochten sind, eine Konstellation, um die sich auch der Laie, besonders aber der Schriftsteller im Hinblick auf mögliche und zum Teil schon eingetretene gefährliche Entwicklungen kümmern muss. Ja, er geht sogar noch weiter, wenn er in seinem Essay «Vom Sinn der Dichtung in unserer Zeit» (1956) fragt, ob nicht die Naturwissenschaft die alte Philosophie des Wortes, wie sie beispielsweise Heidegger vertritt, abgelöst hat und die neue Philosophie bei Einstein und Heisenberg zu finden ist. Erkenntnistheoretisch bleibt F. D. hin- und hergerissen zwischen der platonischen Ideenlehre einerseits und dem hypothetischen Realismus Campbells andererseits, den Konrad Lorenz in seinem Buch «Die Rückseite des Spiegels» vertritt, ohne sich für die eine oder andere Seite endgültig zu entscheiden. Das philosophische Erbe aus seiner Studienzeit scheint die sehr viel später erworbenen biologischen Kenntnisse zu dominieren.

Er widerlegt mit seinem Werk die These von Ch. P. Snow in dessen ironisch-bissigem Essay «The two Cultures», dass eine Verständigung zwischen Geisteswissenschaft und Naturwissenschaft nicht möglich sei, weil die Vertreter der einen Richtung zu eingebildet, die der anderen zu beschäftigt seien. Dürrenmatt vollzieht in seinem Werk die fruchtbare Synthese. 
Wenn es Aufgabe des Schriftstellers und Denkers ist, die Welt und die Zeit, in der wir leben, zu deuten, dann geht das heute nicht mehr ohne die Berücksichtigung der Naturwissenschaften und der daraus abgeleiteten Technik. Zu tief haben sie unser Sein beeinflusst und geprägt. Das setzt allerdings profunde Kenntnisse voraus. «Wir müssen, wollen wir sie beschreiben, unsere Welt verstehen.» ${ }^{128}$ Dürrenmatt hat sich diese Kenntnisse angeeignet und sieht überdies die innigen Verflechtungen der einzelnen Disziplinen über ihre Grenzen hinaus.

128 Interview mit seinem Verleger und Galeristen Daniel Keel, 1978, in: Keel, D.: Über Friedrich Dürrenmatt. Zürich: Diogenes, 1990.

\section{Literatur}

- Arnold, H. L.: Spielen wir zum letzten Mal Komödie. Heinrich-Heine-Institut, Düsseldorf 1993.

- Feynman, R. P.: Vom Wesen physikalischer Gesetze, München/Zürich: Serie Piper, 1993.

- Fischer, E. P.: Eine verschränkte Welt. mannheimer forum 87/88, Boehringer Mannheim $\mathrm{GmbH}$.

- von Glasenapp, H.: Die Religionen Indiens. Stuttgart: Kröner Verlag, 1943.

- Goertz, H.: Dürrenmatt. Reinbek: Rowohlt, 1991.

- Grimm, G. E.: Die Welt als Labyrinth. NZZ Nr. 21 vom 26.1.1994.

- Hawking, St. W.: Eine kurze Geschichte der Zeit. Reinbek: Rowohlt, 1988.

- dto.: Mein Standpunkt. Die Zeit Nr. 34 vom 20. 8. 1993.

- dto.: Was war vor dem grossen Knall? - Spiegel-Interview. Der Spiegel Nr. 42/1988.

- Hieber, J.: König Dürrenmatt. FAZ Nr. 82 vom 9. 4. 1994.

- Kaiser, G.: Viel Gerede, kein Gespräch. Die Zeit vom 18. 3. 1994.

- Krättli, A.: Der Gedankenschlosser an der Arbeit. NZZ Nr. 217 vom 18. 9. 1992.

- Leaky, R./Lewin, R.: Der Ursprung des Menschen. Frankfurt/M.: S. Fischer, 1992.

- Lemke, U.: Gottähnlicher Schöpfer oder biologische Zwischenstation? Süddeutsche Zeiung am Wochenende vom 23.7.1993.

- Lorenz, K./Kreuzer, F.: Leben ist lernen. München/Zürich: R. Piper.

- von Matt, B.: Friedrich Dürrenmatt, der Zeitgemässe. NZZ Nr. 71 vom 14. 3. 1994.

- von Matt, P.: Vom Sonnenschicksal. FAZ Nr. 139 vom 18. 6. 1994.

- Mössbauer, R. L.: Rätselhafte Neutrinos. Vortrag 1992 in Duisburg (Reihe: Nobelpreisträger im Ruhrgebiet).

- Monod, J: Zufall und Notwendigkeit. München: R. Piper, 1971.

- Nachmansohn, D./Schmid, R.: Die grosse Ära der Wissenschaft in Deutschland 1900 bis 1933 , Stuttgart: Wissenschaftliche Verlagsanstalt, 1988.

- Popper, K. R.: Objektive Erkenntnis. Hamburg: Campe paperback, 1993.

- Popper, K. R./ Eccles, J. C.: Das Ich und sein Gehirn. München: Piper, 1982.

- Primas, H.: Ein Ganzes, das nicht aus Teilen besteht. Neue Horizonte 92/93. München: R. Piper.

- Raddatz, F.: im Gespräch mit F. D. Die Zeit vom 16. 8. 1988.

- Schnabel, U.: Krach um den Urknall. Die Zeit Nr. 24 vom 11. 6. 1993.

- Siemes, Ch.: Raumpatrouille Dürrenmatt. Die Zeit Nr. 15 vom 8. 4. 1994.

- Stent, G. S.: Die Autonomie des Menschen. Neue Horizonte 92/93. München: R. Piper.

- Teilhard de Chardin, P.: Der Mensch im Kosmos. München: C. H. Beck, 1959.

- dto.: Die Entstehung des Menschen. München: C. H. Beck, 1961.

- Vollmer, G.: Wissenschaft mit Steinzeitgehirnen? mannheimer forum 86/87, Boehringer Mannheim GmbH.

- Weyl, H.: Philosophie der Mathematik und Naturwissenschaft. München: R. Oldenbourg Verlag, 1990. 